\title{
Extended Hopanoid Loss Reduces Bacterial Motility and Surface Attachment and Leads to Heterogeneity in Root Nodule Growth Kinetics in a Bradyrhizobium-Aeschynomene Symbiosis
}

\author{
Brittany J. Belin, ${ }^{1}$ Elise M. Tookmanian, ${ }^{2}$ Jaime de Anda, ${ }^{3}$ Gerard C. L. Wong, ${ }^{4}$ and Dianne K. Newman ${ }^{1,4, \dagger}$ \\ ${ }^{1}$ Division of Biology \& Bioengineering, California Institute of Technology, Pasadena, CA, U.S.A. \\ ${ }^{2}$ Division of Chemistry \& Chemical Engineering, California Institute of Technology \\ ${ }^{3}$ Department of Bioengineering, Department of Chemistry and Biochemistry, and California NanoSystems Institute, University \\ of California Los Angeles, Los Angeles, CA, U.S.A. \\ ${ }^{4}$ Division of Geological \& Planetary Sciences, California Institute of Technology
}

Accepted 27 May 2019.

\begin{abstract}
Hopanoids are steroid-like bacterial lipids that enhance membrane rigidity and promote bacterial growth under diverse stresses. Hopanoid biosynthesis genes are conserved in nitrogen-fixing plant symbionts, and we previously found that the extended $\left(\mathrm{C}_{35}\right)$ class of hopanoids in Bradyrhizobium diazoefficiens are required for efficient symbiotic nitrogen fixation in the tropical legume host Aeschynomene afraspera. Here, we demonstrate that the nitrogen-fixation defect conferred by extended hopanoid loss can be fully explained by a reduction in root nodule sizes rather than per-bacteroid nitrogen-fixation levels. Using a single-nodule tracking approach to quantify $A$. afraspera nodule development, we provide a quantitative model of root nodule development in this host, uncovering both the baseline growth parameters for wild-type nodules and a surprising heterogeneity of extended hopanoid mutant developmental phenotypes. These phenotypes include a delay in root nodule initiation and the presence of a subpopulation of nodules with slow growth rates and low final volumes, which are correlated with reduced motility and surface attachment in vitro and lower bacteroid densities in planta, respectively. This work provides a quantitative reference point for understanding the phenotypic diversity of ineffective symbionts in $A$. afraspera and identifies specific developmental stages affected by extended hopanoid loss for future mechanistic work.
\end{abstract}

${ }^{\dagger}$ Corresponding author: D. Newman; dkn@ caltech.edu

Funding: This work was supported by grants from Howard Hughes Medical Institute (D. K. Newman), National Aeronautics and Space Administration (NNX16AL96G, D. K. Newman), Jane Coffin Childs Memorial Fund (B. J. Belin), National Institute of General Medical Sciences (K99GM126141, B. J. Belin), and the Army Research Office (W911NF-181-0254, G. C. L. Wong) and predoctoral fellowships from the National Science Foundation (E. M. Tookmanian), and the Ford Foundation (J. de Anda).

*The $\boldsymbol{e}$-Xtra logo stands for "electronic extra" and indicates that 20 supplementary figures, one supplementary table, and four supplementary videos are published online.

The author(s) declare no conflict of interest.

(c) 2019 The American Phytopathological Society
Keywords: rhizobium-legume symbiosis, hopanoids, motility, Aeschynomene, Bradyrhizobium

Hopanoids are steroid-like lipids that support bacterial survival under stress (Belin et al. 2018). They are synthesized by the squalene-hopene cyclase (shc) family of enzymes (Ochs et al. 1992; Syrén et al. 2016), which generate the pentacyclic, $C_{30}$ hopanoid core from squalene. In many organisms, the $\mathrm{C}_{30}$ hopanoids can be further modified, including methylation at the C-2 position via the enzyme HpnP (Welander et al. 2010) and addition of a ribose-derived side chain by the enzyme $\mathrm{HpnH}$ (Fig. 1A) (Welander et al. 2012). Side chain-containing hopanoids are known collectively as the $\mathrm{C}_{35}$ or extended hopanoids and commonly include molecules with aminotriol-, polyol-, and adenosyl-side-chain moieties (Schmerk et al. 2015). Organismspecific side chains have also been observed, including a hopanoid-lipid A conjugate known as HoLA (Komaniecka et al. 2014; Kulkarni et al. 2015; Silipo et al. 2014) that, so far, has only been found in members of the family Bradyrhizobiaceae.

It is thought that hopanoids primarily promote bacterial survival by rigidifying and decreasing the permeability of membranes (Sáenz et al. 2015; Wu et al. 2015), providing a better barrier against external stress. Structurally distinct hopanoids have different capacities to alter the biophysical properties of membranes and can also differ in the degrees of stress resistance they confer (Belin et al. 2018). In the Bradyrhizobium genus of legume symbionts, hopanoids promote growth of free-living cultures under acid, salt, detergent, antibiotic, and redox stresses (Kulkarni et al. 2015; Silipo et al. 2014), and we previously showed that these stress resistance phenotypes are largely mediated by the extended hopanoid class (Kulkarni et al. 2015).

We also analyzed an extended hopanoid-deficient mutant of Bradyrhizobium diazoefficiens USDA110 in symbiosis with two legumes, the native soybean host for this strain, and Aeschynomene afraspera, the native host of the closely related photosynthetic bradyrhizobia. A. afraspera is a flood-tolerant legume from tropical West Africa, where it has been used in rice intercropping systems (Somado et al. 2003) and to accelerate wound healing in traditional medicine (CaamalFuentes et al. 2015; Chifundera 2001; Lei et al. 2019; Swapna et al. 2011). We found that extended hopanoid-deficient 
mutants of $B$. diazoefficiens fixed less nitrogen per nodule in A. afraspera than wild type (WT), while this strain did not appear to have a defect in its native soybean host. Microscopy analyses of a small sample of extended hopanoid mutant-infected $A$. afraspera nodules revealed several aberrant cytological phenotypes, including nodules containing necrotic signatures, disorganized infection zones, and visible starch granule accumulation (Kulkarni et al. 2015).

These phenotypes are common signatures of poor symbiont performance, yet the lack of genetic tools for A. afraspera, the limited literature on this host's response to noncooperators compared with model plants, and the low number of nodules examined made it difficult to determine the underlying cause. While it has been proposed that hopanoids may enable high rates of symbiotic nitrogen fixation in some hosts by limiting oxygen diffusion across cell membranes (Abeysekera et al. 1990; Parsons et al. 1987; Vilcheze et al. 1994), from our previous assays, we could not determine whether the poor symbiotic performance of extended hopanoid mutants reflects ineffective nitrogen fixation per se or is simply a consequence of lower general stress resistance. Because we did not observe an extended hopanoid mutant phenotype in soybean, we instead suggested that the extended hopanoid mutant may not survive exposure to nodule cysteine-rich (NCR) peptides, which are synthesized by $A$. afraspera (Czernic et al. 2015) but absent in soybean.

Here, we sought to dissect further the symbiotic phenotypes of $B$. diazoefficiens extended hopanoid mutants in association with $A$. afraspera. We found that the lower nitrogen fixation of extended hopanoid mutants can be fully explained by a reduction in root nodule sizes and rhizobial occupancy, indicating that the underlying defect is unrelated to per-bacteroid nitrogen fixation levels. Using a novel single-nodule tracking approach to quantify $A$. afraspera nodule development, we uncovered both the baseline growth parameters for WT nodules and a surprising heterogeneity of extended hopanoid
A

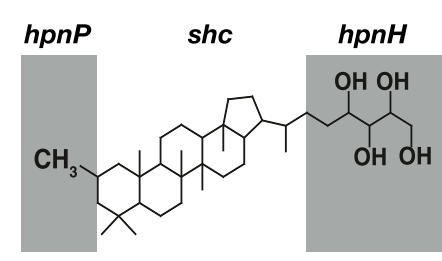

D

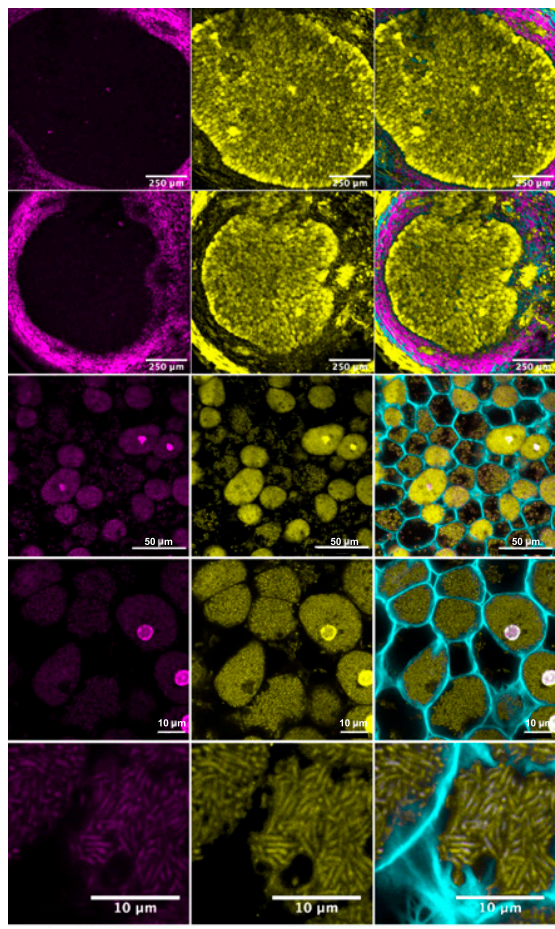

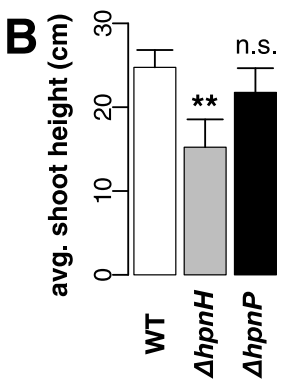
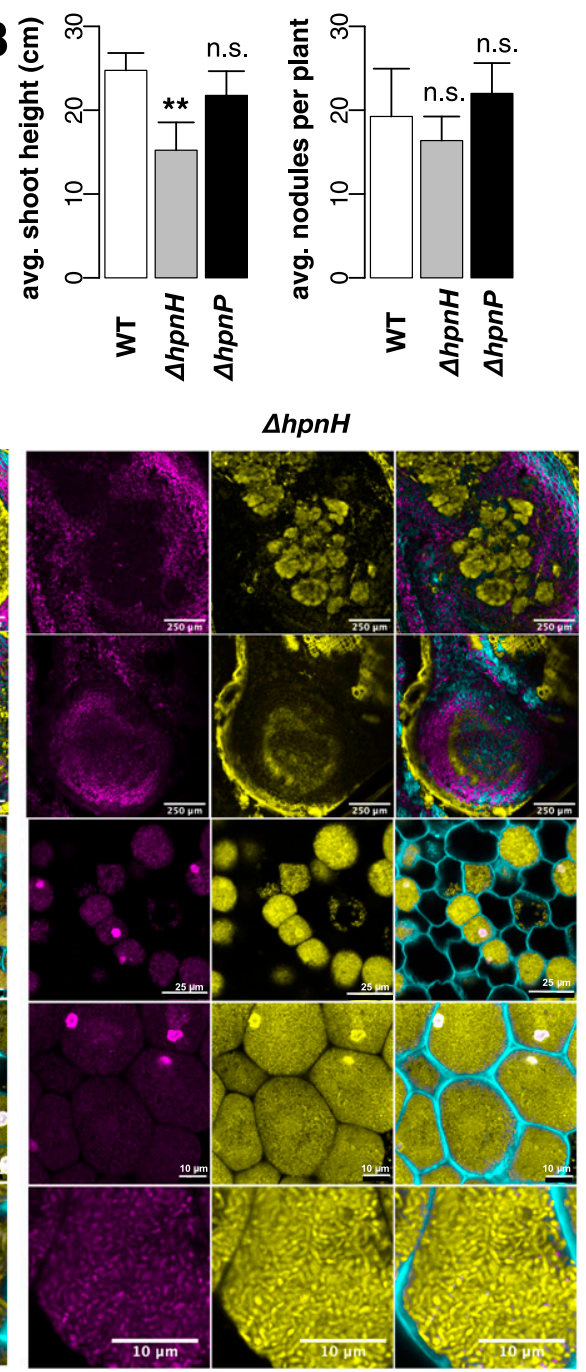

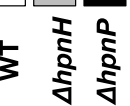
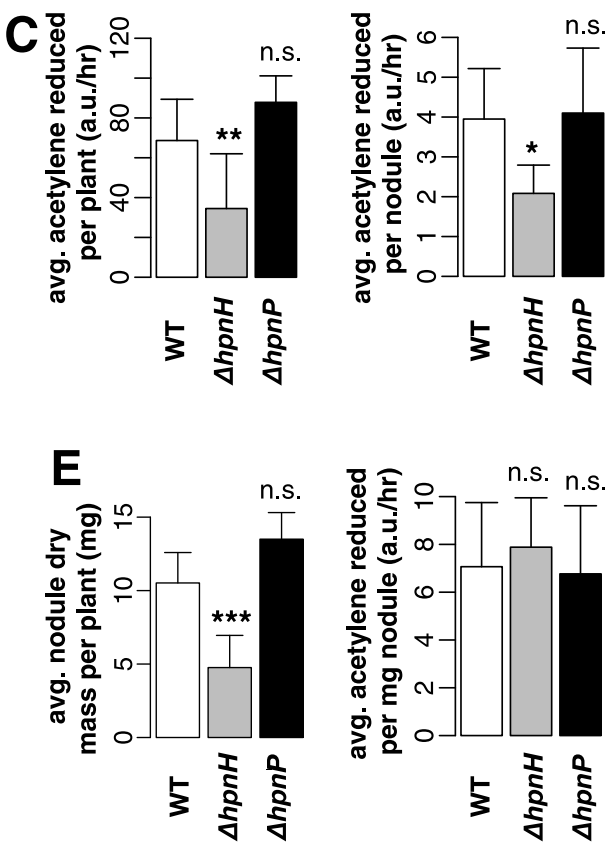

$\mathbf{F}$

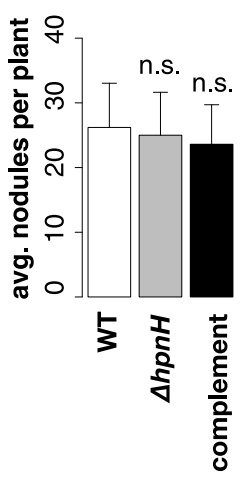

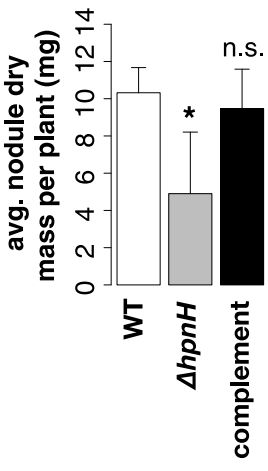

Fig. 1. The nitrogen-fixation defect of $\triangle h p n H$ results from a reduction in nodule sizes. A, Chemical structure of the extended hopanoid 2-methyl bacteriohopanetetrol, consisting of a central pentacyclic core synthesized by the shc gene product, a $C 2$ methylation site added by the product of $h p n P$ (gray shading, left), and a tetrol group added by the $h p n H$ product (gray shading, right). B, Average shoot heights and number of nodules per plant at 24 days postinoculation (dpi) for Aeschynomene afraspera plants inoculated with wild type (WT), $\Delta h p n H$, or $\Delta h p n P$ Bradyrhizobium diazoefficiens. C, Average acetylene reduction per plant and per nodule at $24 \mathrm{dpi}$ for A. afraspera plants inoculated with WT, $\Delta h p n H$, or $\Delta h p n P$. D, Representative confocal images of cross-sections of WT-and $\Delta h p n H$-infected nodules at $24 \mathrm{dpi}$, illustrating plant cell walls (Calcofluor, cyan), live bacteria (SYTO9, yellow), and membrane-compromised bacteria and plant nuclei (propidium iodide, magenta). E, Average nodule dry mass and acetylene reduction per nodule dry mass at 24 dpi for plants inoculated with WT, $\Delta h p n H$, or $\Delta h p n P$. F, Average number of nodules and nodule dry mass at 24 dpi for plants inoculated with WT, $\Delta h p n H$, or a $\Delta h p n H$ complement strain. Data shown in B, C, E, and F were collected from eight plants $(n=8)$, with error bars representing one standard deviation. Results of two-tailed $t$ tests between WT and $\Delta h p n H$ or $\triangle h p n P$ are denoted as follows: n.s., $P>0.05$; one asterisk $\left(^{*}\right), P<0.01$; two $(* *), P<0.001$; and three $(* * *), P<0.0001$. 
mutant developmental phenotypes. These results challenge the conclusions of our prior study (Kulkarni et al. 2015) and identify new, potentially hopanoid-dependent stages in the $B$. diazoefficiens $-A$. afraspera symbiosis for future mechanistic work. This work also provides a quantitative reference point for understanding the impact of symbiotically ineffective strains on $A$. afraspera nodule development.

\section{RESULTS}

Loss of extended hopanoids results in reduced nodule size.

Previously, we observed a symbiotic defect for an extended hopanoid-deficient $(\Delta h p n H)$ strain of $B$. diazoefficiens in association with $A$. afraspera (Kulkarni et al. 2015). To further validate this defect, we inoculated $A$. afraspera plants with $\Delta h p n H$ (lacking extended hopanoids), $\Delta h p n P$ (lacking 2-methyl hopanoids), or WT B. diazoefficiens. At 24 days postinoculation (dpi), plants inoculated with $\Delta h p n H$ were shorter than WT-inoculated plants, although both strains produced equivalent numbers of nodules (Fig. 1B). $\Delta h p n H$-inoculated plants also exhibited a roughly $50 \%$ decrease in the rate of acetylene gas reduction compared with WT-inoculated plants at this timepoint (Fig. 1C). In contrast, the $\Delta h p n P$ mutant was similar to WT (Fig. 1B and C). These results are consistent with our previous findings (Kulkarni et al. 2015).

To assess $\Delta h p n H$ viability within $A$. afraspera nodules, we performed morphological analyses of nodules using confocal fluorescence microscopy. A total of $57 \mathrm{WT}$ and $67 \Delta h p n H$ nodule cross-sections were stained with a bacterial live/dead kit, consisting of the cell-permeable SYTO9 dye (staining all cells) and propidium iodide (PI) (staining only cells with a compromised membrane). We did not observe an increase in predominantly PI-stained nodules for $\Delta h p n H$ compared with WT (Supplementary Figs. S1 and S2). Signatures of plant necrosis, which we previously associated with $\Delta h p n H$ when we observed a smaller number of nodules (Kulkarni et al. 2015), occurred prominently in only one of $67 \Delta h p n H$ nodules examined. However, we did find that many $\Delta h p n H$ nodules contained disorganized infection zones and that $\Delta h p n H$ bacteroids were less elongated than WT (Fig. 1D), as we reported previously (Kulkarni et al. 2015).

The most apparent phenotype of $\Delta h p n H$ nodules was their relatively small size (Fig. 1D). We repeated acetylene reduction assays for WT- and $\Delta h p n H$-inoculated plants and calculated the total nodule dry mass for each plant at $24 \mathrm{dpi}$. We found a decrease in the nodule dry mass per plant for $\Delta h p n H$-inoculated plants that is sufficient to explain the decrease in acetylene reduction rates (Fig. 1E). This decrease in nodule dry mass can be fully rescued by integrating the $h p n H$ gene at the endogenous scoI locus (Fig. 1F), suggesting that the lower nodule mass is due to hpnH loss specifically. This result rules out the possibility that nitrogenase functions ineffectively in the absence of extended hopanoids due to inactivation by oxygen, as has been suggested in Frankia spp. (Abeysekera et al. 1990; Parsons et al. 1987; Vilcheze et al. 1994), as the per-milligram nitrogen fixation rates are not affected by extended hopanoid loss.

\section{$\Delta$ hpnH nodules are more variable in size than WT nodules.}

We measured acetylene reduction per plant across an extended 40-dpi period, and we observed that the differences in both acetylene reduction rates and nodule dry masses between WT and $\Delta h p n H$ steadily decreased with time (Fig. 2A and B). By $40 \mathrm{dpi}$, the overall symbiotic efficiencies of WT and $\Delta h p n H$ per plant were indistinguishable, in terms of plant qualitative appearance (Fig. 2C and D) as well as their average shoot heights and acetylene reduction rates (Supplementary Fig. S3). Total nodule counts per plant also did not differ between WT and $\triangle h p n H$ at $40 \mathrm{dpi}$, indicating that the increase in total nodule mass reflects growing nodules rather than more frequent nodulation.

We also measured the radii of individual nodules on ten plants for each strain at $40 \mathrm{dpi}$ (Fig. 2E and F). Interestingly, although average nodule sizes did become similar between strains by this timepoint ( 0.73 versus $0.88 \mathrm{~mm}$ average radii), their underlying distributions were markedly distinct. Wildtype nodule radii appear to form a roughly normal distribution, whereas the $\Delta h p n H$ nodule radius distribution is bimodal, consisting of a subpopulation of small nodules with small radii $(<0.5 \mathrm{~mm})$ that are rarely observed in WT, as well as a second, larger subpopulation that has a similar median radius as WT but is skewed toward larger radii $(>1.5 \mathrm{~mm})$. These data demonstrate that the small-nodule phenotype of $\Delta h p n H$ persists throughout a 40-dpi time course but is compensated by greater size heterogeneity, in which a handful of 'mega' nodules offset smaller nodules over time.

\section{$\Delta$ hpnH nodule size heterogeneity reflects variable nodule growth rates.}

To better evaluate the possible origins of the $\Delta h p n H$ nodule size defect, we studied the kinetics of single nodule development. Beginning 1 week after inoculation, we collected images
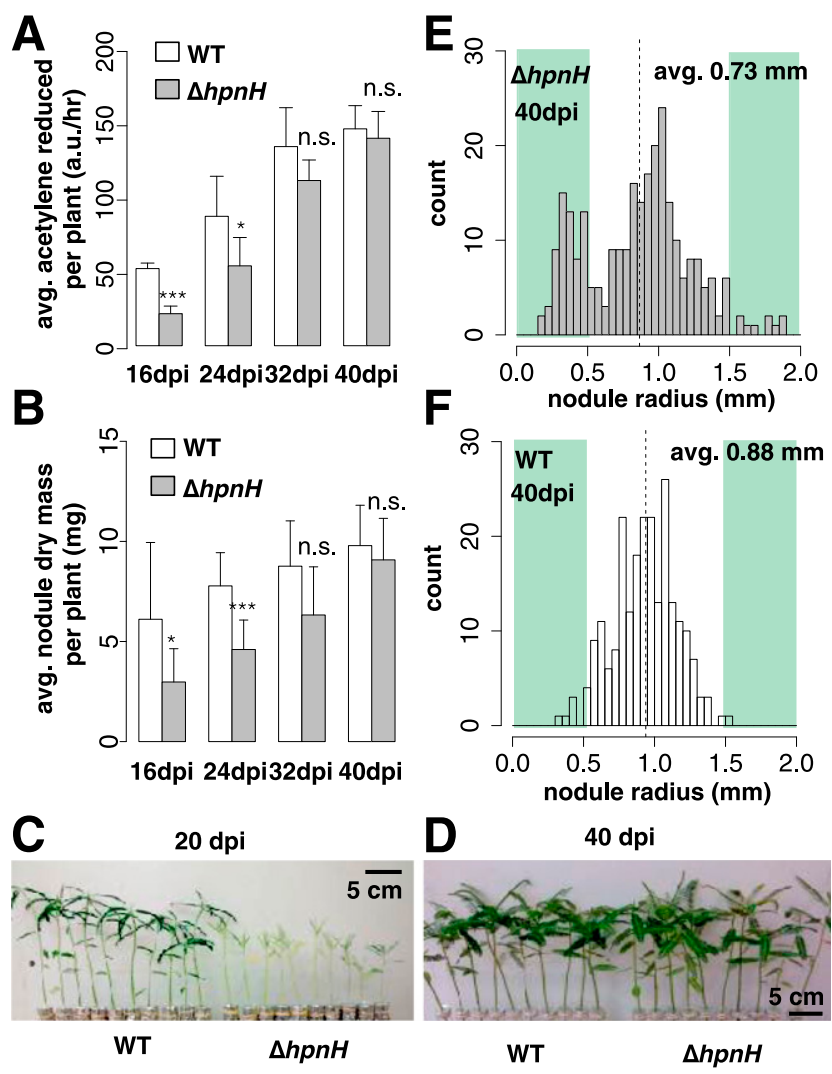

Fig. 2. Smaller $\Delta h p n H$ nodules are offset by increased nodule size heterogeneity over time. A, Average acetylene reduction per plant ( $n=4$ plants per bar) and $\mathbf{B}$, average nodule dry mass per plant ( $n=8$ plants per bar) for A. afraspera inoculated with wild type (WT) or $\Delta h p n H$ over time. Error bars represent one standard deviation. Results of two-tailed $t$ tests between WT and $\Delta h p n H$ are denoted as follows: n.s., $P>0.05$; one asterisk (*), $P<0.05$; three (***), $P<0.0001$. C and D, Aeschynomene afraspera inoculated with WT or $\Delta h p n H$ at 20 days postinoculation (dpi) (C) and 40 dpi (D). E and F, Distributions of nodule diameters at $40 \mathrm{dpi}$ for $A$. afraspera inoculated with $\Delta h p n H$ ( $n=268$ nodules pooled from 10 plants) (E) or WT $(n=227$ nodules pooled from 10 plants) $(\mathrm{F})$. 
of entire plant roots every 3 to 5 days, up to about 40 days postinoculation (Supplementary Figs. S4 and S5). From these images, we identified nodules that were clearly visible (e.g., not obscured by lateral roots or more recently emerged nodules) in at least five timepoints (Fig. 3A) and measured their radii. We calculated nodule volumes by approximating nodules as spheres and plotted the volume of the tracked nodules over time. While we again observed that many $\Delta h p n H$ nodules were smaller at 40 dpi than any of the WT nodules, we also found that nodule growth was highly variable both within and between strains (Fig. 3B and C).

We developed a simple framework for quantifying nodule development, in which nodule growth is defined by the following variables: the time of the initial intracellular infection event $\left(t_{\mathrm{i}}\right)$ and the volume of the nascent nodule $\left(\mathrm{V}_{\mathrm{i}}\right)$, equivalent to the volume of one infected $A$. afraspera cortical cell; the time $\left(t_{\min }\right)$ and volume $\left(\mathrm{V}_{\min }\right)$ at which a clearly visible, spherical nodule has developed; the rate of growth of a nodule once it has become visible $(\mathrm{dV} / \mathrm{d} t)$; and the time $\left(t_{\max }\right)$ and volume $\left(\mathrm{V}_{\max }\right)$ of a nodule when its growth has stopped (Fig. 3D). To calculate these variables, we fit the growth over time of each nodule to three different growth models, exponential, quadratic, and a generalized logistic (e.g., sigmoidal) equation commonly used for plant growth (Richards 1959; Szparaga and Kocira 2018) (complete details below). Sigmoidal models generally provided the best fit to the experimental data, so these models were used for growth parameter calculation (Fig. 3E; Supplementary Figs. S6 and S7).

The growth rates of $\Delta h p n H$ nodules were lower on average than WT nodules (Fig. 3F), with roughly a third of tracked nodules exhibiting growth rates lower than observed for WT $(<0.1$ cubic millimeter per day postinoculation). A similar fraction of nodules had smaller final volumes than WT (Fig. $3 \mathrm{G})$. We further found that the growth rate of a nodule and its maximum size are positively linearly correlated for both strains, with Pearson coefficients of approximately $0.64(P<$ $\left.10^{-9}\right)$ for WT and approximately $0.75\left(P<10^{-15}\right)$ for $\Delta h p n H$, and that the subpopulation of nodules with lower-than-WT growth rates and small nodule sizes are the same (Fig. $3 \mathrm{H})$. We interpret these data to suggest that either host cell proliferation, host cell expansion, or both is slower in a subset of nodules infected with $\Delta h p n H$ and that this largely accounts for the low final volume of these nodules.

We also noted that $\triangle h p n H$ nodule sizes at 40 dpi differed between these single-nodule volume measurements (Fig. 3G) and our previous 40-dpi end-point measurements of nodule radii (Fig. 2E) in that we did not observe larger-than-WT mega nodules in the single-nodule dataset. This discrepancy likely reflects the smaller sample size in our single-nodule tracking experiments ( 84 compared with 268 end-point nodules) and the low frequency of mega nodule formation. To verify this, we selected 10,000 random subsets of 84 nodules from the 268 $\Delta h p n H$ nodules shown in Figure 2E, converted the nodule radii to volumes, and found that there is no statistically significant difference $(P<0.05)$ between a random subset $($ Fig. $2 \mathrm{E})$ and the $\Delta h p n H$ single-nodule tracking data in approximately $92 \%$ $(9,184$ of 10,000$)$ of cases. Thus, the differences in nodule size distributions illustrated in Figures $2 \mathrm{E}$ and $3 \mathrm{G}$ are consistent with sampling error.

We also calculated the window of maximum growth of each nodule, defined as the time required for a nodule to increase from 10 to $90 \%$ of its final volume. Neither the time at which a nodule reaches $90 \%$ of its maximum volume, $t_{\max }$, nor the window of maximum growth differs significantly between $\Delta h p n H$ and WT (Supplementary Fig. S8A and B). The window of maximum growth for each nodule is also uncorrelated with their final volume or growth rate, indicating that small nodules are not prematurely aborted; rather, their growth periods are similar to those of larger nodules (Supplementary Fig. S9A to D).

To better understand the subpopulation of small, slowgrowing $\Delta h p n H$ nodules, we isolated nodules with a radius less than $0.5 \mathrm{~mm}$, sectioned and stained them with SYTO9, PI, and Calcofluor, and imaged them with confocal microscopy. We found that, while most small $\Delta h p n H$ nodules contained a single, continuous infection zone, a large fraction were un- or underinfected with bacteria, often exhibiting disorganized central infection zones ( 28 out of 74 total nodules, approximately $37 \%$ ) (Fig. 4A; Supplementary Fig. S10). Of the fully infected small $\Delta h p n H$ nodules, a subset contained primarily PI-stained, likely dead, bacterial cells (12 of 47 fully infected nodules, approximately 25\%) (Fig. 4A). Similar proportions of underinfected nodules or nodules primarily occupied with membranecompromised bacteria did not occur in larger $\Delta h p n H$ nodules harvested at the same timepoint, although fragmented infection zones were still common (Fig. 4B; Supplementary Fig. S11). We also compared the subpopulation of small $\Delta h p n H$ nodules at 40 dpi to two WT nodule populations: i) similar small nodules harvested at 10 and 25 dpi (Supplementary Figs. S12 and S13), and ii) nodules harvested at the same 40-dpi timepoint (Supplementary Fig. S14). Again, we found that high proportions of underinfected nodules and membrane-compromised bacteria were unique to the $\Delta h p n H$ small-nodule subset.

\section{$\Delta h p n H$ nodule emergence is delayed.}

The 'true' beginning of nodule formation is the time when the first $A$. afraspera cortical cell is infected, i.e., $t_{\mathrm{i}}$ (Fig. 3D). However, this initial infection event is not visible at the root surface, and it is difficult to extrapolate from sigmoidal models in which the growth curves approach the initial volume, $\mathrm{V}_{\mathrm{i}}$ approximately $0 \mathrm{~mm}^{3}$, asymptotically. As a proxy for $t_{\mathrm{i}}$, we defined three alternate $t_{\min }$ as the times at which nodules reached three arbitrarily small volumes: $\mathrm{V}=0.05 \mathrm{~mm}^{3}, \mathrm{~V}=$ $0.1 \mathrm{~mm}^{3}$, and $\mathrm{V}=0.2 \mathrm{~mm}^{3}$. When $t_{\min }$ is defined by $\mathrm{V}=$ $0.05 \mathrm{~mm}^{3}$ or $0.1 \mathrm{~mm}^{3}, t_{\min }$ could not be accurately calculated for all nodules, as the sigmoidal models sometimes predicted an impossible $t_{\min }<0$. These nodule volumes are also too small to be seen on the root surface, and we had no experimental means to determine the accuracy of the calculations in this lowvolume regime. When $t_{\mathrm{min}}$ is defined by $\mathrm{V}=0.2 \mathrm{~mm}^{3}$ (the smallest nodule volume that we could identify in our singlenodule tracking assays), there is a small but statistically significant increase for $\Delta h p n H$ relative to WT.

To independently verify this delay in nodule emergence, we inspected the roots of $20 \mathrm{WT}$ - and $20 \Delta h p n H$-inoculated plants over $40 \mathrm{dpi}$ and recorded the number of visible nodules per plant each day. We found a more even distribution of observed $t_{\min }$ for $\Delta h p n H$ relative to WT, with a 1 to 3 day shift in the most frequent day postinoculation. Surprisingly, we also found that the formation of new nodules is periodic, with a new 'burst' of nodules emerging roughly every 18 days (Fig. 3I). This periodicity of nodule emergence appears to be similar between strains.

While the slight $t_{\min }$ delay for $\Delta h p n H$ is consistent with longer times required to initiate the symbiosis (e.g., rootsurface colonization, invasion of the root epidermis and cortex, and intracellular uptake), it is also possible that a delay in $t_{\min }$ simply reflects a lower rate of nodule growth immediately after the first intracellular infection. To address this, we compared the calculated value of $t_{\min }$ (defined by $\mathrm{V}=0.2 \mathrm{~mm}^{3}$ ) to the maximum growth rates and volumes for each nodule. We did not find that nodules with lower growth rates and final volumes than WT were more likely to have a later $t_{\min }$, supporting the interpretation that the delay in $t_{\min }$ of $\Delta h p n H$ could be due to a 

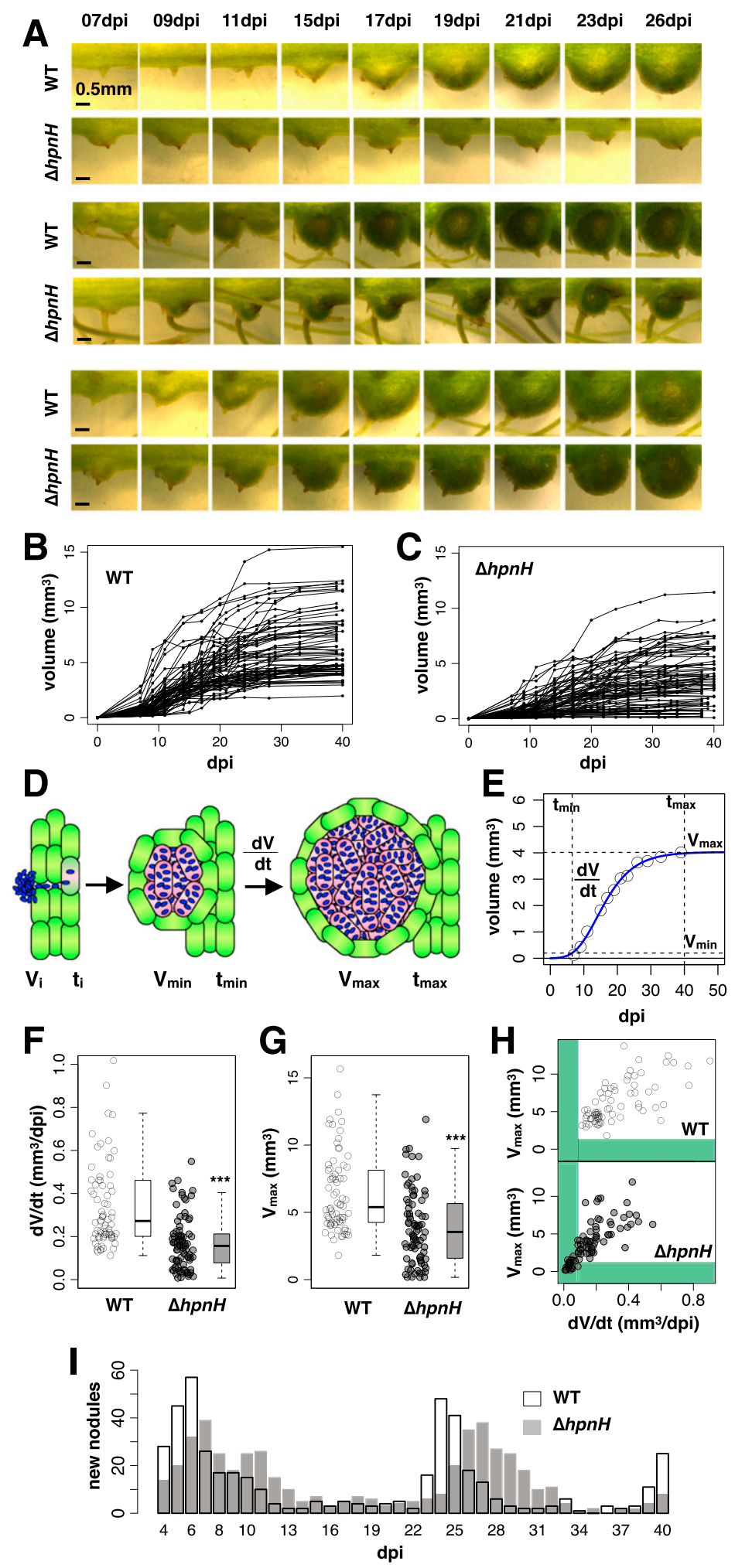

Fig. 3. Nodules containing $\triangle h p n H$ emerge later and have more heterogeneous growth rates and final volumes than wild type (WT). A, Comparison of the development of selected WT- and $\Delta h p n H$-infected nodules over time. B, Nodule growth plots for 74 WT-infected nodules tracked from 10 plants. C, Nodule growth plots for $84 \Delta h p n$-infected nodules tracked from 16 plants. D, Schematic of nodule development in Aeschynomene afraspera. From the left, bacteria (in blue) colonize and invade plant roots (green) and intracellularly infect a root cell (pink); the time of this initial intracellular infection is considered $t_{\mathrm{i}}$, and the nodule volume can be described as the volume of the single infected root cell, $\mathrm{V}_{\mathrm{i}}$. This infected cell proliferates to form a spherical nodule that is visible to the naked eye, at time $t_{\min }$ and volume $\mathrm{V}_{\min }$. The infected plant cells continue to proliferate at rate $\mathrm{dV} / \mathrm{d} t$ until the nodule has fully matured at time $t_{\max }$ and volume $\mathrm{V}_{\max }$. E, Fitted growth curve for a sample WT nodule illustrating the positions of $t_{\min }, \mathrm{V}_{\min }, \mathrm{dV} / \mathrm{d} t, t_{\max }$, and $\mathrm{V}_{\max }$. F and $\mathbf{G}$, Jitter and box plots of dV/d $t(\mathrm{~F})$ and $\mathrm{V}_{\max }$ values $(\mathrm{G})$ for all WT- and $\Delta h p n H$-infected nodules. Results of KS-tests between WT and $\Delta h p n H$ nodules are denoted as follows: three asterisks ( $* * *$ ), $P<10^{-6}$. H, Scatter plots of $\mathrm{dV} / \mathrm{d} t$ versus $\mathrm{V}_{\max }$ values for WT and $\Delta h p n H$ nodules. Values of $\mathrm{dV} / \mathrm{d} t$ and $\mathrm{V}_{\max }$ below what is observed in the WT dataset are highlighted in green. I, Distributions of $t_{\min }$ values (as observed by eye) for nodules from WT-or $\Delta h p n H$-infected plants (white or gray bars, respectively). $n=$ 457 WT nodules across 20 plants and $479 \Delta h p n H$ nodules across 20 plants. 
separate initiation defect. Interestingly, $t_{\min }$ is also not correlated with the period in which maximum nodule growth occurs, such that later-emerging nodules have a growth period similar to nodules formed within a few days postinoculation. This indicates that, although nodule emergence is restricted to narrow, periodic windows (Fig. 3I), once a nodule has entered its maximum growth phase, its continued growth is comparatively unconstrained.

\section{$\Delta h p n H$ is delayed in a pre-endosymbiont stage.}

We performed competition assays using a standard fluorescence labeling approach. We first generated $\Delta h p n H$ and WT strains expressing chromosomally integrated fluorescent proteins, and we coinoculated A. afraspera with different ratios of these two strains. As control experiments, we also coinoculated each tagged strain with its untagged counterpart, in order to determine the effect of fluorescent protein overexpression on the competitiveness of each strain. After $40 \mathrm{dpi}$, we measured the size of nodules on plants inoculated with each strain combination and ratio and sectioned and fixed nodules for imaging. Although we expected each nodule to contain a clonal population of symbionts, based on previous work (Bonaldi et al. 2011; Ledermann et al. 2015), the majority of the nodules instead contained a mixture of both strains (Fig. 5A).

We quantified the relative abundance of each strain in each nodule by fluorescence imaging. In our control experiments, in which only one fluorophore-expressing strain was present, a DNA dye was used to label all bacteria. Both yellow fluorescent protein-tagged WT (WT-YFP) and $\Delta h p n H$-mCherry were significantly out-competed by their corresponding untagged strains. In nodules with higher proportions of tagged strains, we observed lower bacterial DNA abundance and smaller nodule and infection zone sizes (Fig. 5B and C; Supplementary Figs. S16, S17, and S18). Additionally, plants coinoculated with untagged- $\Delta h p n H$ and $\Delta h p n H$-mCherry were significantly shorter than plants inoculated with untagged- $\Delta h p n H$ only, suggesting $\Delta h p n H$-mCherry is symbiotically defective (Supplementary Fig. S15).
These effects of fluorophore overexpression made it difficult to interpret our WT-YFP and $\Delta h p n H$-mCherry competition data, so we developed an alternative, antibiotics-based method to study the timing of early symbiotic initiation. First, we identified antibiotics that were effective against $B$. diazoefficiens but would minimally affect $A$. afraspera growth. We tested three antibiotics (streptomycin at $100 \mu \mathrm{g} / \mathrm{ml}$, kanamycin at $100 \mu \mathrm{g} / \mathrm{ml}$, and tetracycline at $20 \mu \mathrm{g} / \mathrm{ml}$ ) and treated noninoculated plants with these antibiotics for 2 weeks, alone and in combination. After this treatment, we found that neither kanamycin nor streptomycin nor the combination of the two significantly affected plant appearance, shoot height, or root and shoot dry masses compared with untreated controls (Supplementary Fig. S19). Plants treated with tetracycline were noticeably more yellow in color, indicating chlorosis, and the roots and plant medium became brown; these plants also had lower shoot and root dry masses than untreated plants.

Because the $\Delta h p n H$ strain is more sensitive to antibiotics than WT (Kulkarni et al. 2015), we tested various concentrations of the non-plant perturbing antibiotics streptomycin and kanamycin to identify concentrations that would result in the same rates of cell death for both strains. We inoculated plant growth media with WT or $\Delta h p n H$ to the same cell densities and under the same environmental conditions as in plant inoculation experiments. The WT culture was supplemented with $100 \mu \mathrm{g}$ of streptomycin per milliliter plus $100 \mu \mathrm{g}$ of kanamycin per milliliter, and $\Delta h p n H$ cultures were supplemented with decreasing concentrations of these antibiotics: 75, 50, and $25 \mu \mathrm{g} / \mathrm{ml}$ each. Samples of the cultures were collected, were serially diluted, and were added to PSY (Regensburger and Hennecke 1983) plates to estimate CFU per milliliter over time. At $50 \mu \mathrm{g}$ of kanamycin per milliliter plus $50 \mu \mathrm{g}$ of streptomycin per milliliter, the rate of decrease in CFUs per milliliter for $\Delta h p n H$ was equivalent to that of WT treated with $100 \mu \mathrm{g}$ of kanamycin per milliliter plus streptomycin (Fig. 5D).

We inoculated 40 plants, each, with WT or $\Delta h p n H$ and added streptomycin and kanamycin to $100 \mu \mathrm{g} / \mathrm{ml}$ each or $50 \mu \mathrm{g} / \mathrm{ml}$ each, respectively, at various points postinoculation. After
A
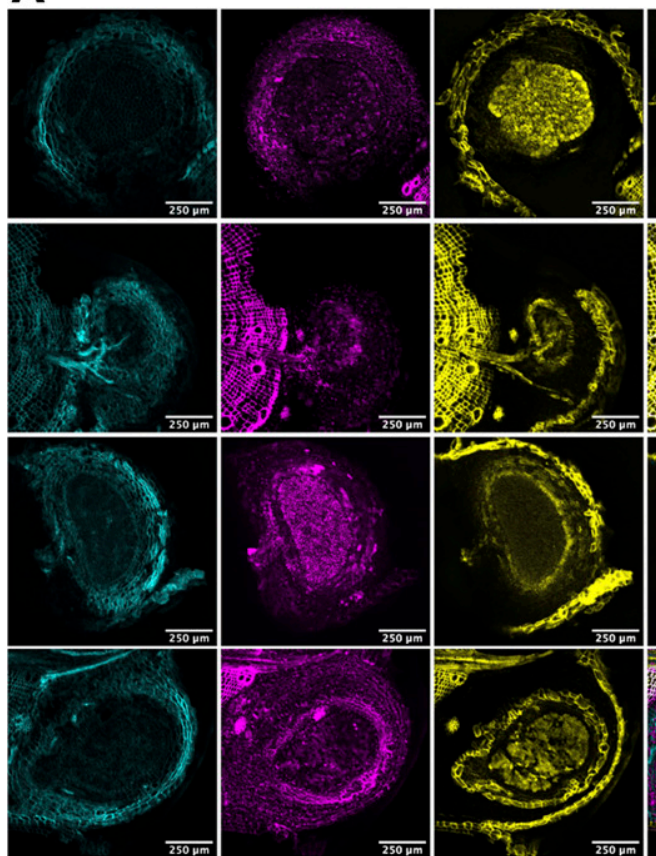
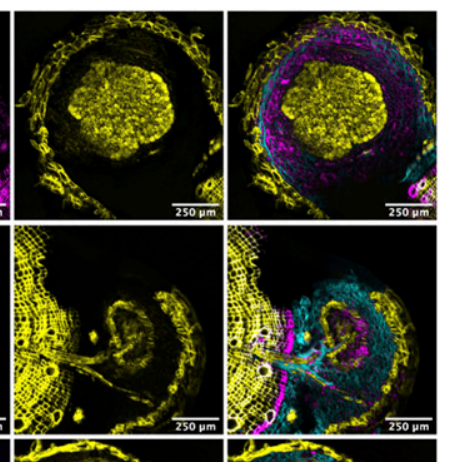

B
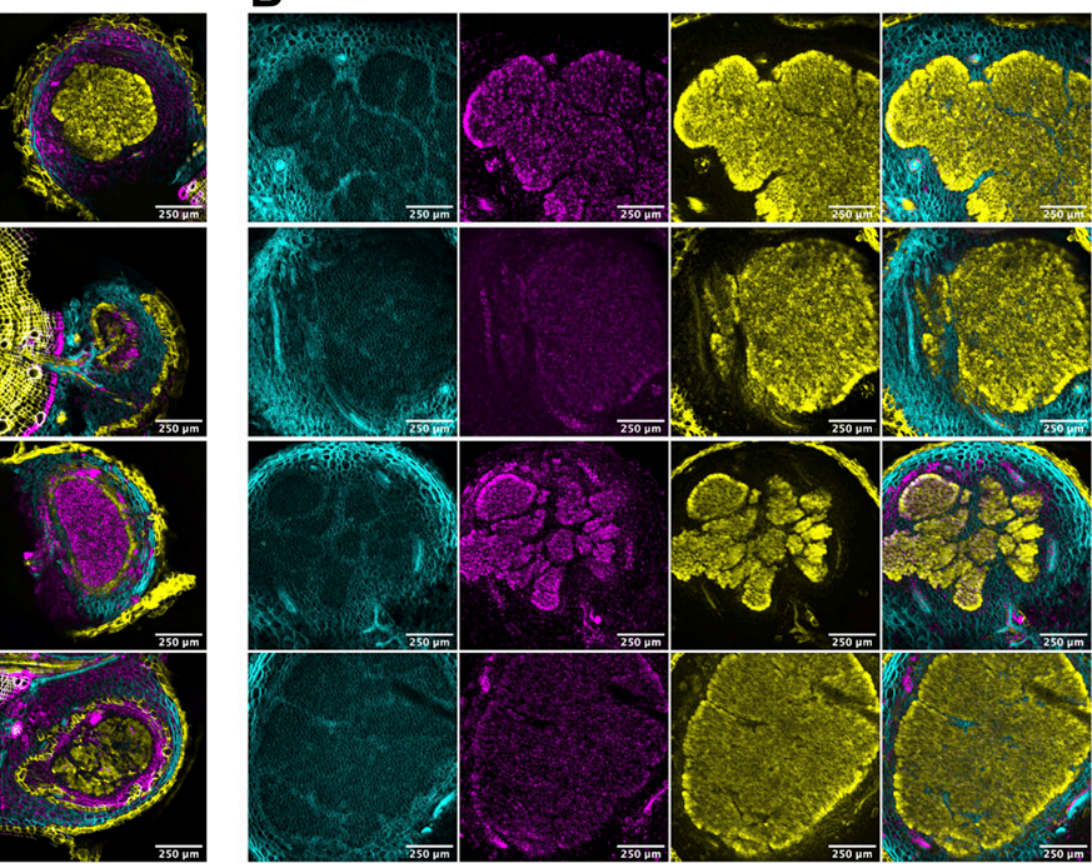

Fig. 4. Small $\Delta h p n H$ nodules are underinfected compared with wild type. A, Confocal sections of small ( $<0.5 \mathrm{~mm}$ radius) $\Delta h p n H$-infected nodules harvested at 40 days postinoculation (dpi). B, Confocal sections of larger ( $>0.5 \mathrm{~mm}$ radius) $\Delta h p n H$-infected nodules harvested at 40 dpi. 
40 days we counted the number of nodules per plant and found that antibiotics were able to block nodule formation over an approximately $50 \%$ longer window in $\Delta h p n H$ compared with WT (Fig. 5E). The decrease in nodules formed at different antibiotic treatment timepoints was also evident in the overall appearance of the plants (Fig. 5F). These results suggest that $\Delta h p n H$ requires more time, on average, to reach the intracellular stage of the symbiosis, at which point, we presume that the bacteria are protected from antibiotic by the host cells. These data would be consistent with $\Delta h p n H$ requiring more time to colonize the root surface, invade the root epidermis, and be internalized by host cells.

\section{Extended hopanoids support surface attachment and motility in vitro.}

Because we found that expression of genetic tags in WT and $\Delta h p n H$ perturbed their symbiosis with $A$. afraspera and because we found that the viability of the hopanoid mutant is reduced by sonication, centrifugation, and mechanical or detergent-based tissue disruption techniques required to reisolate bacteria from plants, we could not directly monitor the colonization of plant roots by these strains.

Instead, we used an in-vitro approach to study the motility and adhesion of these strains on abiotic surfaces. Although these assays do not fully capture the environment of the root surface, defects in abiotic surface attachment can correlate with defects in host colonization (Nagy et al. 2015) and abiotic substrates can be sufficient to elicit some host response genes (Siryaporn et al. 2014). To determine whether $\Delta h p n H$ is less motile than WT, we inoculated low-agar, PSY plates with $\Delta h p n H$ or WT and measured the rate of zone of swimming over time. We observed that the diameter of motility was reduced in $\Delta h p n H$ compared with WT (Fig. 6A and B), consistent with a swimming motility defect; however, because we have previously shown that $\Delta h p n H$ grows more slowly in this medium than WT (Kulkarni et al. 2015), we could not rule out the possibility that slower zone expansion simply reflects a longer doubling time.

To investigate the nature of the plate motility defect, we studied the motility of single $B$. diazoefficiens cells. We inoculated cells into glass-bottom, sterile PSY flow cells with $100 \mu \mathrm{l}$ of each strain and recorded the movement of cells near the glass surface at $5 \mathrm{~ms}$ time resolution. Trajectories of individual motile cells, defined as cells having superdiffusive motion and a trajectory radius of gyration $>2.5 \mu \mathrm{m}$, were calculated and analyzed in MATLAB (Lee et al. 2018). In agreement with results from motility plate assays, we observed significantly fewer $(P<0.0001)$ motile cells in $\Delta h p n H$ cultures
A

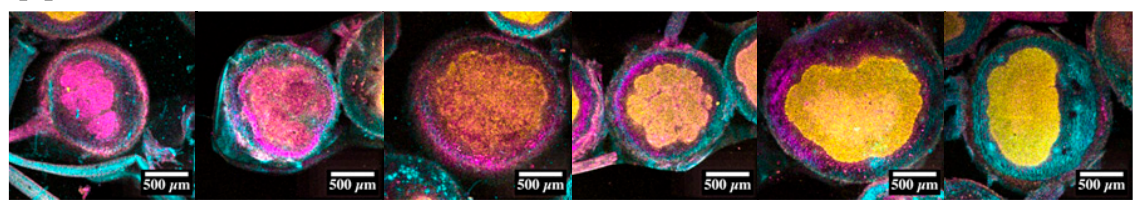

B

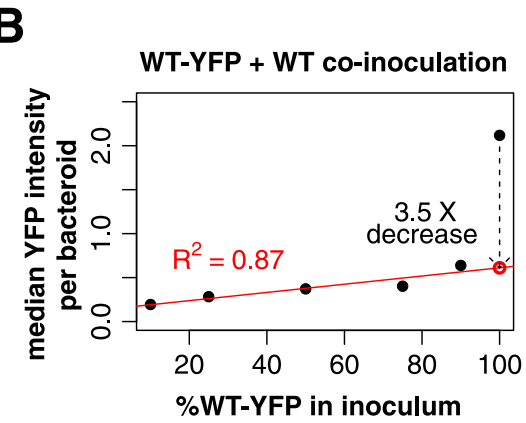

D

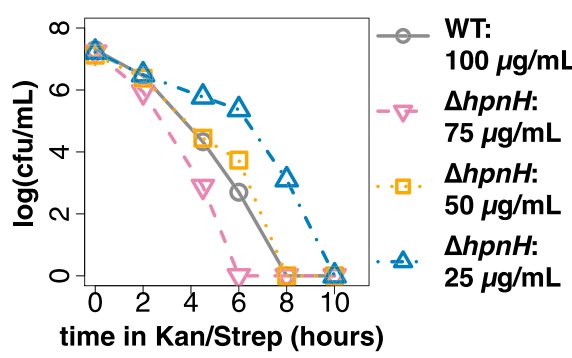

C

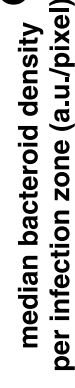

WT-YFP + WT co-inoculation

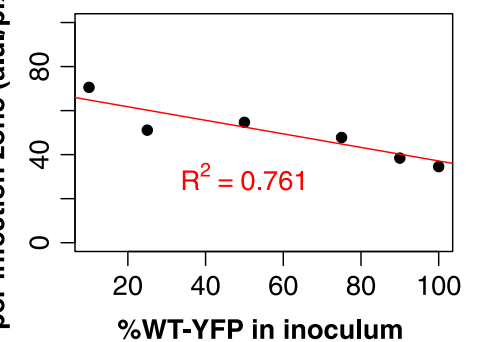

F

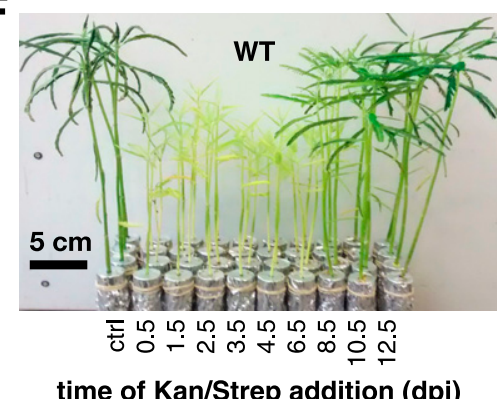

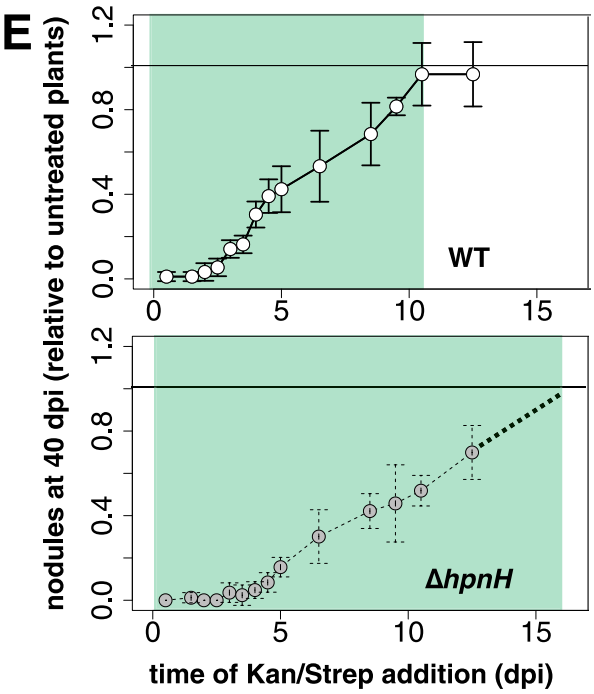

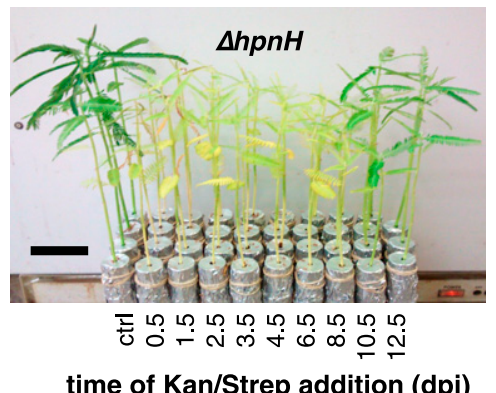

Fig. 5. Extended hopanoid mutants are delayed at pre-intracellular stages in symbiosis development. A, Confocal sections of nodules from plants coinoculated with wild type tagged with yellow fluorescent protein (WT-YFP) and $\Delta$ hpn $H$-mCherry harvested at 45 to 55 days postinoculation (dpi). Sections were stained with Calcofluor (cyan) and are expressing YFP (yellow) and mCherry (magenta). B, Scatter plot of median YFP intensity per pixel normalized by propidium iodide (PI) intensity per pixel (e.g., bacteroid density) within infection zones of nodules from plants coinoculated with WT-YFP and WT, as a function of the percentage of WT-YFP in the inoculum. C, Scatter plot of median PI intensity per pixel (e.g., bacteroid density) within infection zones of nodules from plants coinoculated with WT-YFP and untagged WT, as a function of the percentage of WT-YFP in the inoculum. D, Wild type and $\Delta h p n H$ viability (measured in CFU per milliliter) grown in buffered nodulation medium supplemented with varying concentrations of kanamycin and spectinomycin at various times after treatment. E, Average nodules per plant at $40 \mathrm{dpi}$ for plants inoculated with either WT or $\Delta h p n H$ and treated with (per milliliter) $50(\Delta h p n H)$ or $100 \mu \mathrm{g}(\mathrm{WT})$ of kanamycin and streptomycin at various timepoints postinoculation. Nodule counts are normalized to those observed in non-antibiotic treated plants. F, Images of inoculated plants at $40 \mathrm{dpi}$ after antibiotic treatment at various timepoints. Untreated plants are shown on the left, with increasing time of antibiotic addition. Error bars represent one standard deviation. 
$(n=65 \pm 29)$ compared with WT cultures $(n=368 \pm 60)$ when cells were grown and assayed in PSY medium (Fig. 6C and D; Supplementary Table S1). Among the motile cells in each population, average mean speeds did not differ significantly $(P>0.05)$, with $<\mathrm{V}>_{\Delta \mathrm{hpnH}}=24.83 \pm 7.0 \mu \mathrm{m} / \mathrm{s}$ and $<\mathrm{V}>_{\mathrm{wt}}=$ $22.75 \pm 6.7 \mu \mathrm{m} / \mathrm{s}$. We repeated these assays in plant growth medium (buffered nodulation medium [BNM]) supplemented with arabinose and ammonia. Under this condition, we again observed a lower fraction of motile $\Delta h p n H$ cells than WT cells $\left(\mathrm{N}_{\Delta \mathrm{hpnH}}=54 \pm 59\right.$ and $\mathrm{N}_{\mathrm{wt}}=450 \pm 310, P<0.01$ ) (Fig. $6 \mathrm{E}$ and F). The mean speeds among motile cells grown and assayed in BNM were also similar between strains, with $\langle\mathrm{V}\rangle_{\Delta \mathrm{hpnH}}=$ $25.04 \pm 6.6 \mu \mathrm{m} / \mathrm{s}$ and $\langle\mathrm{V}\rangle_{\mathrm{wt}}=22.99 \pm 6.4 \mu \mathrm{m} / \mathrm{s}$, and did not differ significantly $(P>0.05)$.

We tested the surface attachment capabilities of $\Delta h p n H$ and WT by incubating dense bacterial cultures on glass coverslips and quantifying the fraction of the surface covered with stably adherent cells after $2 \mathrm{~h}$. In PSY medium, both strains adhered poorly and there was no significant difference in their attachment efficiencies (Fig. 6G; Supplementary Fig. S20). In BNM supplemented with arabinose and ammonia, both strains adhered to glass better than in PSY and $\Delta h p n H$ attachment levels were significantly lower than WT (Fig. 6H). Because a decrease in stably adherent cells and in motile cells within $\Delta h p n H$ cultures could reflect a lower number of viable cells in this strain, we also measured CFUs per milliliter in WT and $\Delta h p n H$ cultures grown to varying cell densities (optical density at $600 \mathrm{~nm}\left[\mathrm{OD}_{600}\right]$ of 0.2 to 1.0 ) in either PSY or BNM supplemented with arabinose and ammonia. We did not find differences in the CFUs per milliliter in each strain for any of the cell densities and medium conditions tested (Fig. 6I), demonstrating that reduced in-vitro adhesion and motility among $\Delta h p n H$ cells cannot be attributed to higher levels of cell death. The decreased adhesion and reduced motile cell population of $\Delta h p n H$ suggest that stable root colonization by this strain may be less efficient, although we cannot account for possible differences in adhesion mechanisms used during attachment to glass versus attachment to the plant root surface.

\section{DISCUSSION}

Hopanoids are well-established mediators of bacterial survival under stress and, previously, we showed that the capacity for hopanoid production is enriched in plant-associated environments (Ricci et al. 2014) and required for optimal Bradyrhizobia-Aeschynomene spp. symbioses (Kulkarni et al. 2015; Silipo et al. 2014). Here, we performed a detailed, quantitative evaluation of the extended hopanoid phenotypes in the Bradyrhizobium diazoefficiens-Aeschynomene afraspera symbiosis. We determined that extended hopanoid mutants fix
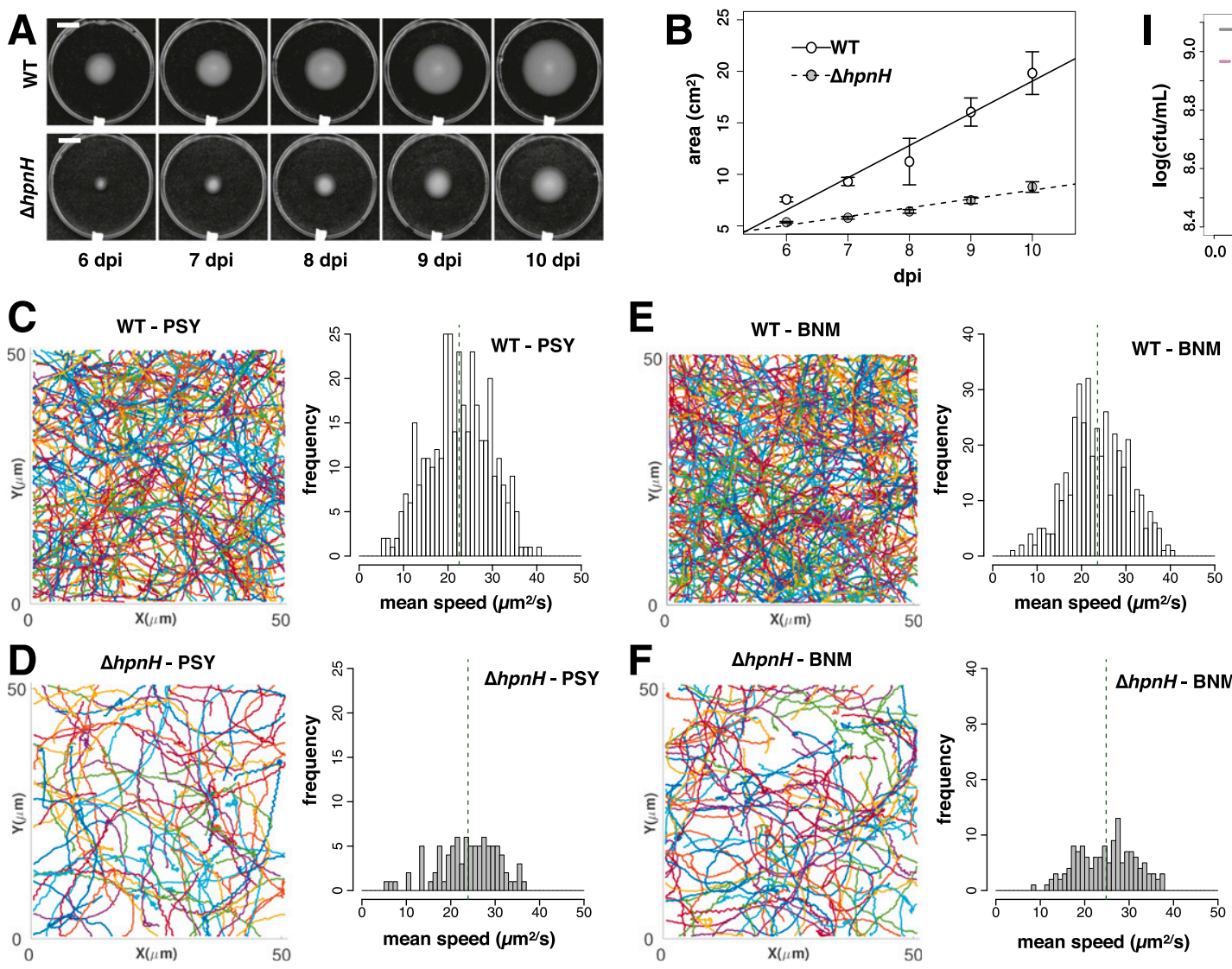
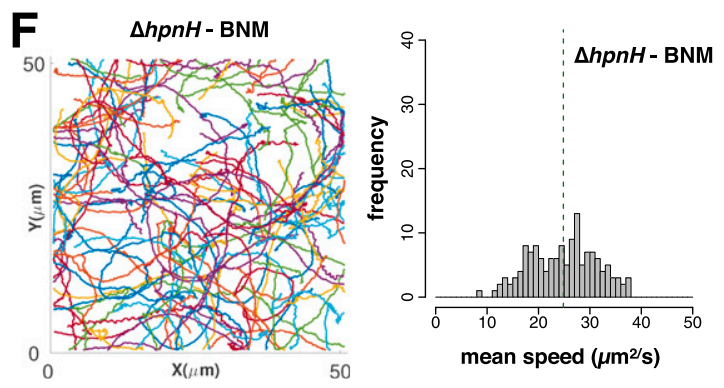

G
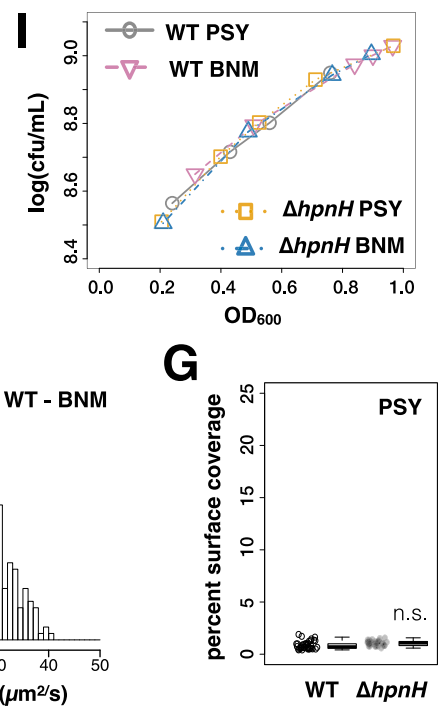

- BNM

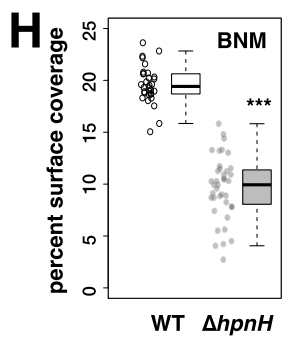

Fig. 6. Extended hopanoid mutants are less motile than wild type (WT) and attach poorly to surfaces in vitro. A, Sample time course of WT and $\Delta h p n H$ colony expansion on low-agar PSY (Regensburger and Hennecke 1983) plates (dpi = days postinoculation). Scale bars represent $2 \mathrm{~cm}$. B, Average colony sizes of WT and $\Delta h p n H$ over time ( $n=4$ plates per strain); error bars indicate one standard deviation. C, Mean speed distribution $(n=359)$ and trajectories for motile WT cells observed over a 5-min time course in PSY. D, Mean speed distribution $(n=91)$ and trajectories for motile $\Delta h p n H$ cells observed over a 5-min time course in PSY. E, Mean speed distribution $(n=421)$ and trajectories for motile WT cells observed over a 5-min time course in buffered nodulation medium $(\mathrm{BNM}) . \mathbf{F}$, Mean speed distribution $(n=141)$ and trajectories for motile $\Delta h p n H$ cells observed over a 5 -min time course in BNM. Vertical dotted lines in the histograms shown in $\mathrm{C}$ to $\mathrm{F}$ indicate the distribution means. $\mathbf{G}$ and $\mathbf{H}$, Jitter and box plots of surface attachment (e.g., the percent of the field of view covered with cells) of WT and $\Delta h p n H$ after $2 \mathrm{~h}$ of incubation on glass in PSY (G) or BNM (H), $n=40$ fields of view per condition. Results of two-tailed $t$ tests between WT and $\Delta h p n H$ are denoted as follows: n.s., $P>0.05$; three asterisks ( $* * *), P<0.00001$. I, WT and $\Delta$ hpnH cultures (measured in CFU per milliliter) grown to a range of $\mathrm{OD}_{600}$ in $\mathrm{BNM}$ supplemented with arabinose and ammonia or in PSY. 
nitrogen at similar rates as WT on a per-bacteroid level, demonstrating that, in this host, extended hopanoids are not required to protect nitrogenase from oxygen, as often has been speculated (Belin et al. 2018). Instead, we found that the lower in-planta productivity of extended hopanoid mutants can be fully attributed to changes in the kinetics of nodule development. By tracking the development of individual root nodules, we observed later nodule emergence times in $\Delta h p n H$ inoculated plants. In vitro, $\Delta h p n H$ cells adhered poorly to glass and were less motile than WT, and it is possible that $\Delta h p n H$ cells are similarly deficient in motility and adhesion in the context of plant association, leading to slower attachment to plant root surfaces (Fig. 7A and B). However, while slower root attachment could explain the later emergence times of $\Delta h p n H$ nodules, more experiments will be needed to determine whether our in-vitro results are relevant to the native Bradyrhizobium-Aeschynomene association.

Through our developmental tracking, we also found that a third of $\Delta h p n H$ nodules grew significantly slower than WT and were smaller at maturity. Many of these small nodules contained low symbiont densities; a subset of larger $\Delta h p n H$ nodules also had lower symbiont loads, due to infection zone fragmentation. The origin of this underinfection is unclear. It is possible that bacteria are inefficiently internalized or retained and this phenotype is simply propagated as nodules develop (Fig. 7C and D). Alternatively, low symbiont densities may reflect symbiont degradation in a previously fully infected nodule (Fig. 7E), perhaps correlating with elicitation of a plant defense response.

These observations challenge two conclusions from our previous work, requiring a refinement of our interpretation of the roles of extended hopanoids in the plant context (Kulkarni et al. 2015). First, we reported that there was no symbiotic defect of the $\Delta h p n H$ strain in soybean, based on the observation that nitrogen fixation per milligram of nodule dry weight was similar to WT. Given that this study revealed that a reduction in nodule dry weight explains the $\Delta h p n H$ defect in A. afraspera, it is possible that this strain is also defective in soybean but this defect was obscured by differences in normalization between the soybean and $A$. afraspera datasets. Second, the majority of $\Delta h p n H$ nodules in A. afraspera had WT-like growth kinetics and morphologies, with a few mega nodules displaying unusually fast growth. This finding appears inconsistent with an inability to survive $A$. afraspera NCR peptides, unless NCR peptide expression levels in $A$. afraspera are extremely variable from nodule to nodule or if the mechanisms that compensate for extended hopanoid loss are stochastic.

What other mechanisms might underpin these extended hopanoid mutant phenotypes? Perhaps they are simply consequences of less rigid $B$. diazoefficiens membranes. The fraction of motile cells in Escherichia coli populations has been suggested to be sensitive to changes to the mechanical properties of the outer membrane (Gupta et al. 2006), and membrane-based mechanotransduction is required by diverse bacteria to stimulate extracellular matrix production and cement their attachment to surfaces (Persat 2017; Petrova and Sauer 2012). B. diazoefficiens mutants with weakened cell walls also have been shown to be deficient in symbiosis with A. afraspera through an NCR peptide-independent mechanism (Barrière et al. 2017), which may be elicited by $\Delta h p n H$. Alternatively, extended hopanoid loss may have secondary effects on Bradyrhizobium-Aeschynomene signaling. In the Frankia-actinorhizal symbiosis, bacterial extended hopanoids can contain the auxinomimetic compound phenyl-acetic acid (Hammad et al. 2003) and, though the effects of hopanoid loss on the bacterial metabolome have not been examined, changes in hopanoid production may impact, one or both, the synthesis and secretion of symbiotically active compounds. Future work will be required to determine whether changes in signaling or membrane mechanics dominate the hopanoid mutant phenotypes and at which developmental stages.

Regardless of the underlying mechanism, it is curious that the absence of extended hopanoids is not a death knell for the $B$. diazoefficiens-A. afraspera symbiosis at any stage, given that, in our previous work (Kulkarni et al. 2015), the $\Delta h p n H$ mutant failed to grow anoxically and exhibited extremely slow growth under acidic and high osmolarity media conditions presumed to mimic the nodule microenvironment. In our invitro studies, mean speeds among motile $\Delta h p n H$ cells were
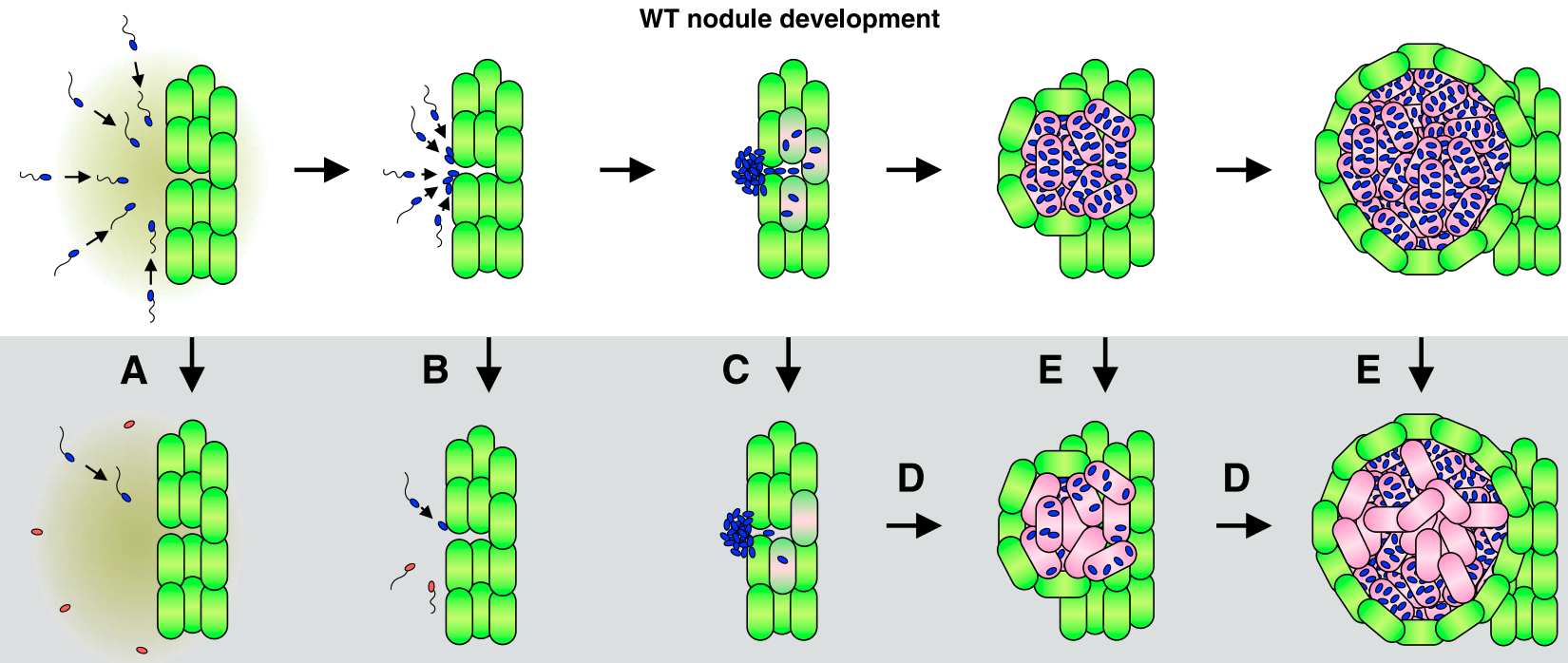

$\Delta h p n H$ symbiotic defects

Fig. 7. Consequences of extended hopanoid loss in Aeschynomene afraspera nodule development. Schematic representation of A. afraspera wild-type root nodule development (top row; white background) and defects in development associated with extended hopanoid loss (bottom row; gray background). A, Early in development, fewer $\Delta h p n H$ cells are motile and $\mathbf{B}$, competent to attach to root surfaces, leading to a delay in establishment of stable root colonies. $\mathbf{C}$, At later stages, slow growth of $\Delta h p n H$ into the root interior or poor uptake by and division within host cells may generate $\mathbf{D}$, 'patchy' or an underpopulated infection zone that is propagated as the nodule grows. E, Alternately, fully infected $\Delta h p n H$ nodules may lose symbionts to symbiont cell death via poor bacteroid survival or plant-directed symbiosome degradation. 
indistinguishable from WT and, though we cannot rule out more subtle defects in the direction of movement or chemotaxis, this suggests that motility systems of $\Delta h p n H$ cells function properly once induced. Similarly, in planta, $\Delta h p n H$ nodules developing at WT rates and reaching average WT volumes did occur and, in the case of mega nodules, some exceeded their WT counterparts. These apparent discrepancies in the in-vitro and in-planta phenotypes of $\Delta h p n H$ suggest that limited inferences can be made between culture models of nodule growth conditions and the in-planta performance of a strain.

Why do $\Delta h p n H$ populations form two distinct populations (WT-like or defective) rather than falling on a continuous distribution of behavior? Bimodality can reflect switch-like or threshold-based regulation, and perhaps, in the $\Delta h p n H$ strain, a fraction of cells cannot support levels of signaling above the threshold required for proper function. Nodules may also differ in the extent to which extended hopanoid loss is compensated. In Methylobacterium extorquens and Rhodopseudomonas palustris (Bradley et al. 2017; Neubauer et al. 2015), hopanoid loss results in upregulation of other membrane-rigidifying lipids, including carotenoids and cardiolipins, and in other plantmicrobe systems, lipid exchange between hosts and microbes has been observed (Keymer and Gutjahr 2018), suggesting that $\Delta h p n H$ nodule phenotypes may relate to the local availability of structurally or functionally similar metabolites. Because of these diverse possible explanations for $\Delta h p n H$ heterogeneity, a detailed comparison of WT-like and defective nodules, including the distributions of lipids and other metabolites, bacteroid morphology and penetrance, and gene expression variability, will be required to determine why some $\Delta h p n H$ nodules succeed and others do not.

Beyond hopanoids, our results provide insight into the developmental control of nodule formation by A. afraspera hosts. We find that nodulation occurs in bursts separated by fixed 18-day intervals and that the timing of these bursts is unrelated to net fixed nitrogen production across the root, more likely reflecting the inherent dynamics of the underlying signaling networks. The growth period of individual nodules is similarly deterministic, suggesting that A. afraspera hosts do not respond to ineffective symbionts by prematurely aborting nodule development. Rather, we find that $A$. afraspera nodules can be primarily distinguished by their growth rates, e.g., the frequencies of infected host cell division and expansion. This finding suggests that, in A. afraspera, host cell mitosis and symbiont performance may be coupled, enabling future studies on the molecular signals through which this coupling occurs.

Finally, our results underscore the importance of identifying the most informative, least perturbing tools for interrogating legume-microbe symbiosis. Employing quantitative, timeresolved, single-nodule and single-cell approaches rather than bulk measurements were essential for uncovering the diverse phenotypes of the $B$. diazoefficiens extended hopanoid mutants and yielded unexpected information on regulation of nodule development by A. afraspera. We have also shown the limitations of introducing overexpressed genetic tags into bacteria. While use of these tags has undoubtedly enhanced our understanding of legume-microbe symbiosis (Ledermann et al. 2018), they may not fully capture the behavior of native organisms. Additionally, our work is one of many to emphasize the importance of appropriate culture models for mimicking the host environment, as the $\Delta h p n H$ surface attachment defect was observed in plant growth medium but not in a standard richer medium. A more detailed analysis of the host environment, including the full milieu of root exudates (Sugiyama and Yazaki 2012), available carbon sources (Pini et al. 2017), and trace metals specific to each legume, will improve in-vitro models of legume-bacteria interactions and may allow selection of strains with improved performance in agriculture.

\section{MATERIALS AND METHODS}

\section{B. diazoefficiens culture and strain generation.}

B. diazoefficiens hopanoid biosynthesis mutants were generated previously (Kulkarni et al. 2015). For construction of YFP- and mCherry-expressing strains, fluorophore expression vectors pRJPaph-YFP and pRJPaph-mCherry (Ledermann et al. 2015) were provided as a gift from H.-M. Fischer (ETH Zurich, Switzerland). For complementation of $\Delta h p n H$ with the endogenous $h p n H$ gene on the strong Paph promoter of pRJPaphmCherry, mCherry was replaced with Paph-hpnH. These vectors were introduced into $B$. diazoefficiens by conjugation with the $\beta 2155$ diaminopimelic acid (DAP) auxotroph strain of $E$. coli, using the following protocol. B. diazoefficiens WT and $\Delta h p n H$ were grown in $5 \mathrm{ml}$ of PSY medium (Regensburger and Hennecke 1983 ) at $30^{\circ} \mathrm{C}$ and $250 \mathrm{rpm}$ to an $\mathrm{OD}_{600}$ of approximately 1.0 (WT) or of 0.5 to $0.8(\Delta h p n H)$. $\beta 2155$ strains carrying pRJPaph vectors were grown to an $\mathrm{OD}_{600}$ of 0.5 to 0.8 in $5 \mathrm{ml}$ of Luria Bertani (LB) supplemented with $10 \mu \mathrm{g}$ of tetracycline per milliliter and $300 \mu \mathrm{m}$ DAP at $37^{\circ} \mathrm{C}$ and $250 \mathrm{rpm}$. Both $B$. diazoefficiens and $\beta 2155$ donor cultures were pelleted at $3,250 \times g$ for $30 \mathrm{~min}$, were washed three times in $0.9 \%$ sterile saline, and were resuspended in $0.9 \%$ sterile saline to a final $\mathrm{OD}_{600}$ of 1.0. B. diazoefficiens strains and $\beta 2155$ donor cells were combined at a 4:1 ratio, respectively, and were mixed by repeated pipetting. Aliquots $(50 \mu \mathrm{l})$ of these $4: 1$ mixtures were dropped to PSY plates supplemented with $300 \mu \mathrm{m}$ DAP, were dried in a biosafety cabinet, and were incubated for $48 \mathrm{~h}$ at $30^{\circ} \mathrm{C}$. Conjugation pastes were removed from plates and were resuspended in $5 \mathrm{ml}$ of sterile saline, were pelleted at $3,250 \times g$ for $30 \mathrm{~min}$, and were washed twice, in order to remove residual DAP. Washed cells were pelleted a final time and were resuspended to $200 \mu \mathrm{l}$ in $0.9 \%$ sterile saline and were plated onto PSY plates supplemented with $20 \mu \mathrm{g}(\mathrm{WT})$ or $10 \mu \mathrm{g}(\Delta h p n H)$ of tetracycline per milliliter. Colonies appeared after 7 to 10 days (WT) or 10 to 14 days $(\Delta h p n H)$, were streaked onto fresh PSY/tetracycline plates, and were sequenced to verify insertion of the pRJPaph vectors into the scoI locus.

\section{A. afraspera cultivation and inoculation with B. diazoefficiens.}

A. afraspera seeds were obtained as a gift from the laboratory of E. Giraud (LSTM/Cirad, Montpelier, France). Seeds were sterilized and scarified by incubation in $95 \%$ sulfuric acid at room temperature (RT) for $45 \mathrm{~min}$, followed by five washes in sterile-filtered Nanopure water and a second incubation in $95 \%$ ethanol for $5 \mathrm{~min}$ at RT. After ethanol treatment seeds were washed five times and were incubated overnight in sterile filtered Nanopure water. Seeds were transferred to freshly poured water/agar plates using sterile, single-use forceps in a biosafety cabinet and were germinated for 24 to $72 \mathrm{~h}$ in the dark at 28 to $32^{\circ} \mathrm{C}$

Seedlings were placed in clear glass test tubes containing $100 \mathrm{ml}$ of sterile, carbon- and nitrogen-free BNM (Ehrhardt et al. 1992) and were grown for 7 to 10 days in plant growth chambers (Percival) under the following settings: $28^{\circ} \mathrm{C}, 80$ to $90 \%$ humidity, and 16-h photoperiod under photosynthetic light bulbs (General Electric) emitting approximately 4,000 lumens per square foot. In parallel, $B$. diazoefficiens strains were grown in 5 to $10 \mathrm{ml}$ of PSY liquid culture at $30^{\circ} \mathrm{C}$ and $250 \mathrm{rpm}$ to stationary phase $\left(\mathrm{OD}_{600}>1.4\right)$. Stationary phase cultures were diluted into PSY 1 day prior to plant inoculation, to reach an $\mathrm{OD}_{600}$ of approximately 0.8 at the time of inoculation. Cultures 
at an $\mathrm{OD}_{600}$ of approximately 0.8 were pelleted at $3,250 \times g$ for $30 \mathrm{~min}$ at RT, were washed once in PSY, and were resuspended in PSY to a final $\mathrm{OD}_{600}$ of 1.0. Resuspended B. diazoefficiens cultures were directly inoculated into the plant medium in a sterile biosafety cabinet, and $1 \mathrm{ml}$ of culture at $\mathrm{OD}_{600}=1.0$ was added per plant. Inoculated plants were returned to growth chambers and were maintained for the times indicated for each experiment. For longer experiments (lasting longer than approximately $30 \mathrm{dpi}$ ), plant growth tubes were refilled with sterile filtered Nanopure water as needed. To minimize cross-contamination, inoculated plants and noninoculated plants were cultivated in separate growth chambers and growth chambers were sterilized with $70 \%$ ethanol followed by UV irradiation for at least $24 \mathrm{~h}$ between experiments.

\section{Acetylene reduction experiments.}

Individual plants were transferred to clear glass $150-\mathrm{ml}$ Balch-type anaerobic culture bottles containing $15 \mathrm{ml}$ of BNM medium and were sealed under a gas-tight septum. After sealing, $15 \mathrm{ml}$ of headspace gas $(10 \%$ of the culture bottle volume) was removed and replaced with $15 \mathrm{ml}$ of acetylene gas (Airgas). Plants in culture bottles were incubated in the light at $28^{\circ} \mathrm{C}$ in growth chambers for 3 to $6 \mathrm{~h}$. A 100 - $\mu$ l sample of the headspace gas was removed using a gastight syringe (Hamilton), and this sample was injected and analyzed for ethylene signal intensities, using a Hewlett Packard 5890 Series II GC with Hewlett Packard 5972 Mass Spectrometer with a $30 \mathrm{~m} \times 0.320 \mathrm{~mm}$ GasPro column (Agilent Technologies) and a 2-mm ID splitless liner (Restek Corporation). Following acetylene reduction measurements, plants were removed from jars and plant shoot heights and number of nodules per plant were recorded. When nodule dry mass measurements were performed, nodules were harvested with a razor blade, were transferred into preweighed Eppendorf tubes, were dried at $50^{\circ} \mathrm{C}$ for a minimum of $48 \mathrm{~h}$, and were weighed again.

\section{Live:Dead staining and imaging of nodule cross-sections.}

Nodules were hand-sectioned with razor blades or on a Leica VT1200 vibratome and were immediately transferred into a fresh solution of $5 \mu \mathrm{M}$ SYTO9 (diluted 1:100 from a $500 \mu \mathrm{M}$ stock in dimethyl sulfoxide at $-20^{\circ} \mathrm{C}$ [Thermo Fisher]) and $0.02 \mathrm{mg} / \mathrm{ml}(30 \mu \mathrm{M})$ PI (diluted 1:50 from a $1 \mathrm{mg} / \mathrm{ml}$ stock stored in water at $4^{\circ} \mathrm{C}$ [Thermo Fisher]) in phosphate buffered saline (PBS). Nodule sections were incubated in this SYTO9/PI solution at RT for $30 \mathrm{~min}$ in the dark with gentle shaking, were washed five times in PBS, and were fixed in $4 \%$ paraformaldehyde (Electron Microscopy Sciences) in PBS overnight in the dark at $4^{\circ} \mathrm{C}$. Fixed sections were washed five times in PBS and were transferred to a freshly prepared solution of $0.1 \mathrm{mg} / \mathrm{ml}$ Calcofluor White (Fluorescence Brightener 28 [Sigma]) in PBS. The sections were incubated in the Calcofluor solution in the dark for $1 \mathrm{~h}$ at RT with gentle shaking and were washed five times in PBS to remove excess dye.

Prior to imaging, sections were transferred to $30-\mathrm{mm}$ imaging dishes with 20-mm \#0 coverglass bottoms (MatTek) and were overlaid with sterile $50 \%$ glycerol. Nodule images were collected on either a Leica TCS SPE laser-scanning confocal (model DMI4000B-CS) using a $10 \times / 0.3$ NA APO ACS objective (for low-resolution images) or a Zeiss LSM 880 laser-scanning confocal equipped with a Fast Airyscan superresolution module using a $63 \times / 1.25$ NA Plan-Neofluar objective (for high-resolution images). Fluorophore excitation was performed at the following settings for each dye: Calcofluor, $405 \mathrm{~nm}$ excitation/410 to $500 \mathrm{~nm}$ emission; SYTO9, $488 \mathrm{~nm}$ excitation/510 to $570 \mathrm{~nm}$ emission; PI, $532 \mathrm{~nm}$ excitation/600 to $650 \mathrm{~nm}$ emission. These images were processed to enhance brightness and contrast in FIJI (Schindelin et al. 2012; Schneider et al. 2012).

\section{Nodule diameter and volume measurements.}

Inoculated $A$. afraspera root nodules were imaged using a high-definition Keyence VHX-600 digital microscope at 20x magnification. For end-point root nodule volume measurements at $40 \mathrm{dpi}$, plants were removed from the growth chamber, were imaged at RT on paper towels, and were discarded. Nodule diameters were measured using the line tool in FIJI and were recorded using a custom FIJI macro. For tracking nodule volumes over time, plants were serially removed from their growth chambers and were transferred to a plastic dish containing $150 \mathrm{ml}$ of sterile $\mathrm{BNM}$ prewarmed to $28^{\circ} \mathrm{C}$. Images of sections of the plant root were collected serially from the hypocotyl to the root tip. Following collection of images, plants were immediately returned to their original growth tubes in the growth chamber. Plastic dishes were sterilized for $10 \mathrm{~min}$ in $10 \%$ bleach, were washed three times in sterile filtered Nanopure water, were sprayed with $70 \%$ ethanol/water, and were air-dried before each new plant was imaged. A fresh aliquot of sterile, prewarmed BNM also was used for each plant. After the time course was completed, images of entire plant root systems were reconstructed by eye for each plant at each timepoint. For nodules appearing in at least five timepoints, nodule diameters were measured as described for the end-point measurements and were converted to approximate volumes in $\mathrm{R}$ using the equation $V=4 / 3 \pi r^{3}$.

\section{Nodule growth curve fitting and analysis.}

All analyses of nodule growth, and corresponding plots, were generated in R. For nodule growth curve fitting, three model equations were used to identify the best fit, as follows:

i) exponential function:

$$
V=a e^{-b t}+c
$$

ii) quadratic function:

$$
V=a t^{2}+b t+c
$$

iii) generalized logistic function (expressed as a Richard's function with a time shift):

$$
V=\frac{a}{\left(1+e^{-b(t-c)}\right)^{\left(\frac{1}{d}\right)}}
$$

Calculation of the optimal parameter values for each equation (e.g., the values of $a, b, c$, and $d$ ) and the standard error for each curve compared with the raw data were performed using the built-in function $n \operatorname{lm}()$ in R. In some cases, $n \operatorname{lm}()$ could not produce a best-fit model without specifying initial values for the function parameters. For exponential models, an equation of best fit could be successfully determined without specification of initial values for parameters $a, b$, and $c$. For quadratic models, initial parameter values were required and were set to $a=0, b=10$ and $c=$ 0 for each nodule plot, after identifying these initial parameter values as broadly optimal based on an initial parameter sweep of -50 to 50 for each plot. For sigmoidal models, no broadly optimal initial values could be identified, so a parameter sweep was performed for each plot with the initial value of $a$ set to the maximum observed nodule volume (as $a$ describes the upper asymptote of the sigmoidal curve), $b$ ranging from 0.1 to $1, c$ ranging from 0 to 10 , and $d$ ranging from 0.01 to 1.0 . In the sigmoidal plots, an initial point of 
$(0,0)$ was added to the nodule volume time series to improve fitting.

Because the sigmoidal model provided the best fits, extrapolation of nodule growth characteristics was performed on sigmoidal models only. The maximum nodule volume, $\mathrm{V}_{\max }$, is defined as the upper asymptote of the sigmoidal growth curve, e.g., $a$. Nodule initiation time, $t_{\mathrm{min}}$, was defined in three separate ways: the times at which the nodule volume is equal to $0.05,0.1$, or $0.2 \mathrm{~mm}^{3}$ (e.g., through solving $0.05,0.1$, or $0.2=$ $a /\left(\left\{1+e^{[-b(t-c)]}\right\}^{(1 / d)}\right)$ for $\left.t\right)$. The maximum nodule growth rate, $\mathrm{dV} / \mathrm{d} t$, was defined as the average rate of growth (e.g., slope) between the time at which the volume is $10 \%$ of $\mathrm{V}_{\max }$ and the time at which the volume is $90 \%$ of $\mathrm{V}_{\max }$. The time at which each nodule reaches its maximum size, $t_{\max }$, was approximated as the time at which the volume is $90 \%$ of $\mathrm{V}_{\max }$, since the true maximum volume is asymptotic to the growth curve and is therefore never fully reached in the model.

\section{Competition assays.}

mCherry-tagged $\Delta h p n H$ and YFP-tagged WT B. diazoefficiens were grown to stationary phase $\left(\mathrm{OD}_{600}>1.4\right)$ in $10 \mathrm{ml}$ of PSY cultures supplemented with 20 (WT) or $10 \mu \mathrm{g}$ per milliliter $(\Delta h p n H)$ tetracycline; untagged strains were grown in PSY. On the day prior to inoculation, all strains were diluted into 50 to $150 \mathrm{ml}$ of tetracycline-free PSY to reach an $\mathrm{OD}_{600}$ of approximately 0.8 at the time of inoculation. A. afraspera plants were cultivated preinoculation in test tubes as described above, with the addition of covering the growth tubes in foil to minimize the production of chlorophyll in the plant roots, which spectrally overlaps with mCherry. At the time of inoculation, all cultures were pelleted at $3,250 \times g$ for $30 \mathrm{~min}$ at RT, were washed three times, and were resuspended in PSY to a final $\mathrm{OD}_{600}$ of 1.0. A $10-\mathrm{ml}$ culture of each strain ratio for inoculation was generated a sterile 15-ml Falcon tube; for example, for a 50:50 mixture of mCherry-tagged $\Delta h p n H$ and YFP-tagged WT, $5 \mathrm{ml}$ of each strain was combined. These cultures were mixed thoroughly by gentle pipetting, and $1 \mathrm{ml}$ of the mixtures was added to directly to the plant medium for seven to eight plants per strain mixture.

After 45 to 60 days, plants were harvested. First, plant heights and the number of nodules per plant were recorded. The roots were cut from the stem and images of all nodules for each plant were collected on a high-definition Keyence VHX-600 digital microscope at $20 \times$ magnification. These nodules were cross-sectioned and were immediately transferred to Eppendorfs containing 4\% paraformaldehyde (Electron Microscopy Sciences) in PBS. Fresh sections were fixed overnight in the dark at $4^{\circ} \mathrm{C}$, were washed five times in PBS, and were stored in PBS supplemented with $0.1 \%$ azide in the dark at $4^{\circ} \mathrm{C}$ until imaging.

Fixed sections were stained in Calcofluor (all strain combinations), PI (WT-YFP and WT coinoculation only), or SYTO9 (mCherry- $\Delta h p n H$ and $\Delta h p n H$ coinoculation only) as described for Live:Dead staining. Imaging was performed as described for Live:Dead staining, using a $5 \times$ objective. Given the high autofluorescence of these nodules and low mCherry and YFP signal intensities, the following excitation/emission settings were used: Calcofluor, $405 \mathrm{~nm}$ excitation/410 to $460 \mathrm{~nm}$ emission; YFP/SYTO9, $488 \mathrm{~nm}$ excitation/500 to $550 \mathrm{~nm}$ emission; and mCherry, $532 \mathrm{~nm}$ excitation/600 to $650 \mathrm{~nm}$ emission.

Quantification of nodule statistics (including nodule and infection zone areas, signal intensity of YFP, mCherry, SYTO9, and PI) was performed on raw images using a custom FIJI macro. Briefly, nodule images were opened at random, infection zones and whole nodules were circled by hand and were saved as discrete regions of interest (ROIs), and the area and intensity in each channel were measured automatically for all ROIs. These measurements were exported as a text table and various parameters from these measurements were calculated, using custom Python scripts. Plots of all parameters and statistical comparisons were generated using custom R scripts. All custom scripts and data used for this analysis are available upon request.

\section{Antibiotic treatment of inoculated plants.}

A. afraspera plants were cultivated as described above and the following antibiotics were added to noninoculated plants 7 days after rooting in $100 \mathrm{ml}$ of BNM growth tubes: kanamycin to $100 \mu \mathrm{g} / \mathrm{ml}$, streptomycin to $100 \mu \mathrm{g} / \mathrm{ml}$, tetracycline to $20 \mu \mathrm{g} / \mathrm{ml}$, kanamycin plus tetracycline, kanamycin plus streptomycin, streptomycin plus tetracycline. Plants were grown in antibiotics under normal plant growth conditions for 14 days, after which plants were visually inspected. Plant heights were also recorded, and the root and shoot systems were separated with a razor blade, were transferred into preweighed $15-\mathrm{ml}$ Falcon tubes, were dried at $50^{\circ} \mathrm{C}$ for a minimum of $48 \mathrm{~h}$, and were weighed again.

Antibiotic treatments of $\Delta h p n H$ and WT B. diazoefficiens were performed by growing antibiotic-free 5-ml PSY cultures of each strain to stationary phase $\left(\mathrm{OD}_{600}>1.4\right)$ and diluting strains in fresh PSY to reach an $\mathrm{OD}_{600}$ of approximately 0.8 at the time of antibiotic treatment-e.g., as they would be grown prior to plant inoculation. Cultures were pelleted at 3,250 $\times g$ for $30 \mathrm{~min}$ at RT, were washed three times, and were resuspended in PSY to a final $\mathrm{OD}_{600}$ of 1.0. Four 100- $\mu \mathrm{l}$ aliquots of these culture were diluted 1:00 into separate $10 \mathrm{ml} \mathrm{BNM}$ cultures in clear glass tubes in plant growth chambers. Kanamycin (at 25, 50, 75, and $100 \mu \mathrm{g} / \mathrm{ml}$ ) and streptomycin (at 25, 50, 75, and $100 \mu \mathrm{g} / \mathrm{ml}$ ) were added directly to the BNM cultures, and 100- $\mu$ l samples were taken immediately prior to antibiotic treatment and at 2, 4, 6, 8, and $10 \mathrm{~h}$ after addition of antibiotics. These 100- $\mu \mathrm{l}$ samples were immediately diluted $1: 10$ in $900 \mu \mathrm{l}$ of $0.9 \%$ sterile saline and were mixed vigorously by repeated pipetting. Vortexing was avoided, as we found that this method reduces $\Delta h p n H$ viability. Ten serial 1:10 dilutions were performed, and three 10- $\mu 1$ samples of each dilution for each strain were spotted and dripped across PSY plates. After 7 (WT) or 10 days $(\Delta h p n H)$, colonies were counted manually and the number of colonies were recorded for each dilution exhibiting discrete colonies. Log plots of colony counts over time were generated in R.

Plants were inoculated with $\Delta h p n H$ and WT B. diazoefficiens as described above, and kanamycin and streptomycin were added to $\triangle h p n H$-inoculated plants to $50 \mu \mathrm{g} / \mathrm{ml}$ each, and to WTinoculated plants to $100 \mu \mathrm{g} / \mathrm{ml}$ at 12 and $36 \mathrm{~h}$ and at 2, 2.5, 3, $3.5,4,4.5,5,6.5,8.5,9.5,10.5$, and 12.5 dpi. Four plants were treated per timepoint per strain, with an additional four plants each as an untreated control. At $40 \mathrm{dpi}$, the number of nodules per plant was recorded.

\section{Bulk motility assays.}

Swimming motility assays were performed as previously described, with some modifications (Althabegoiti et al. 2008). WT and $\triangle h p n H$ were grown to turbidity in $5 \mathrm{ml}$ of PSY at $30^{\circ} \mathrm{C}$ and $250 \mathrm{rpm}$, were diluted to an $\mathrm{OD}_{600}$ of 0.02 in $5 \mathrm{ml}$ of fresh PSY, and were grown to exponential phase $\left(\mathrm{OD}_{600}=0.3\right.$ to 0.5$)$. Exponential cultures were diluted to an $\mathrm{OD}_{600}$ of 0.06 in fresh PSY and $2 \mu \mathrm{l}$ of the adjusted cultures into the center of a swimming plate containing $0.3 \%$ agar/PSY. After inoculation, the plates were wrapped with parafilm to prevent dehydration and were incubated in a humidity-controlled environmental chamber (Percival) at $30^{\circ} \mathrm{C}$ for 10 days total, with daily scans after 5 days. The resulting images were analyzed in FIJI to measure the area of the swimming colony.

\section{Surface attachment assays.}

$\Delta h p n H$ and WT B. diazoefficiens were grown in 5-ml PSY cultures to stationary phase $\left(\mathrm{OD}_{600}>1.4\right)$ and were diluted in 
fresh PSY to reach an $\mathrm{OD}_{600}$ of approximately 0.8 at the time of surface attachment assays. Cultures were pelleted at $3,250 \times g$ for $30 \mathrm{~min}$ at RT, were washed twice in the indicated attachment medium, and were resuspended in attachment medium to an $\mathrm{OD}_{600}$ of 1.0 . These cultures were mixed thoroughly by repeated pipetting, and $2-\mathrm{ml}$ samples were added to sterile imaging dishes (30-mm dishes with $20-\mathrm{mm}$, \#1.5 coverglass bottoms [MatTek]). Cultures were incubated on imaging dishes without shaking at $30^{\circ} \mathrm{C}$ for $2 \mathrm{~h}$. To remove nonadhered cells, imaging dishes were immersed in $50 \mathrm{ml}$ of attachment media in a 100-ml glass beaker on an orbital shaker and were shaken gently at RT for 5 min; direct application of washing medium to the coverglass surface was avoided, as we found that this creates a shear force sufficient to wash away adhered cells. Imaging dishes were gently lifted out of the washing medium and were imaged with a $100 \times$ objective on a Lumascope 720 fluorescence microscope (Etaluma). Forty fields of view were recorded for each strain and media combination. These images were processed in FIJI, using the enhanced local contrast plugin (Heckbert 1994), and were converted into a binary image to determine the area of the imaging window covered with adhered cells. Calculation of the fraction of the surface was performed in Excel and statistical analyses were conducted in R. Areas of the surface containing groups of cells larger than $10 \mu \mathrm{m}^{2}$ in area were ignored in the calculations, as these likely do not represent true attachment events rather than sedimentation of larger cell clumps. BNM used for attachment assays was prepared as described above, with the addition of $1.0 \mathrm{~g}$ of arabinose per milliliter. Because BNM contains salt crystals that can sediment onto coverglass and occlude or obscure adhered cells, this medium was passed through a $2-\mu \mathrm{m}$ filter (Millipore) prior to the attachment experiments.

\section{Single-cell motility assays and analysis.}

B. diazoefficiens WT and $\Delta h p n H$ were grown in $12.5 \mathrm{ml}$ of PSY medium at $30^{\circ} \mathrm{C}$ and $200 \mathrm{rpm}$ to an $\mathrm{OD}_{600}=0.6$ to 0.8 from a PSY medium plate culture. A 1:10 dilution of cell culture was subcultured in PSY medium to a final volume of $12.5 \mathrm{ml}$ and was regrown to an $\mathrm{OD}_{600}$ of approximately 0.6. Two aliquots of $750 \mu \mathrm{l}$ were sampled from the regrowth culture and were pelleted at $3,500 \times g$ for 20 (WT) or for $30 \mathrm{~min}$ $(\Delta h p n H)$ at RT. The supernatant was removed, and one pellet was resuspended in $500 \mu \mathrm{l}$ of PSY and the other in $500 \mu \mathrm{l}$ of BNM medium. Because BNM contains salt crystals that can sediment onto coverglass and occlude or obscure adhered cells, this medium was passed through a $2 \mu \mathrm{m}$ filter (Millipore) prior to usage for these experiments. The two medium conditions were incubated for 2.5 (WT) or $3.5 \mathrm{~h}(\Delta h p n H)$ at $30^{\circ} \mathrm{C}$; given the difference in growth time $\Delta h p n H$ was incubated for longer. Right before imaging, each culture was diluted at a 1:10 ratio with its respective medium. The bacteria were injected into a sterile flow cell (Ibidi sticky-Slide VI0.4 with a glass coverslip). The flow cell was attached to a heating stage set to $30^{\circ} \mathrm{C}$.

The imaging protocol involved high-speed bright-field imaging for 5 min at a single XYZ location per experimental repeat. High speed bright-field recordings used a Phantom V12.1 high speed camera (Vision Research); images were taken with a 5 -ms exposure at $200 \mathrm{fps}$ and a resolution of $512 \times 512$ pixels $(0.1 \mu \mathrm{m} / \mathrm{pixel})$. This protocol was performed on an Olympus IX83 microscope equipped with a $100 \times$ oil objective, a $2 \times$ multiplier lens, and a Zero Drift Correction autofocus system. The recorded movies were extracted into single frames from the .cine files using PCC 2.8 (Phantom Software). Image processing and cell tracking algorithms are adapted from previous work (Lee et al. 2018) and were written in MATLAB R2015a (Mathworks).
We identified cells swimming near the surface as cells with a trajectory radius of gyration greater than $2.5 \mu \mathrm{m}$ and a meansquared displacement (MSD) slope greater than 1.5. Setting a minimum radius of gyration selects for cells with a minimum net translation on the across the surface, while a minimum MSD slope threshold ensured the cells are moving superdiffusively (MSD slope $\cong 1$, diffusive motion; MSD slope $\cong 2$, superdiffusive motion). For each tracked cell, the mean-speed, $v$, was calculated by averaging a moving window, $w$, of the displacement over the cell's full trajectory, using the following equation:

$$
v=A v g\left(\sum_{t=1}^{N-w} \frac{\sqrt{\left(x_{t+w}-x_{t}\right)^{2}+\left(y_{t+w}-y_{t}\right)^{2}}}{w} * f * p\right)
$$

where $N$ is the total number of points in the trajectory, $f$ is the acquisition frame rate, and $p$ is the pixel resolution. Here, we set a window size, $w=40$ frames. All analysis and visualizations from these experiments where done using MATLAB R2015a (Mathworks).

\section{ACKNOWLEDGMENTS}

We thank E. Giraud for his generous gift of $A$. afraspera seeds and training on Aeschynomene symbioses and H.-M. Fischer and R. Ledermann for plasmids and technical advice for the genetic transformation of B. diazoefficiens. N. Dalleska of the Environmental Analysis Center at Caltech was instrumental in providing training and support for gas chromatography mass spectrometry analysis of acetylene reduction. Nodule sectioning was performed using CLARITY, Caltech Optogenetics and Vector Engineering Research (CLOVER) Center, and high-resolution imaging of bacteroids was performed at the Caltech Beckman Imaging Facility, with training from G. Spigolon. We are grateful to G. Kulkarni and other members of the Newman lab as well as to E. Meyerowitz and R. Phillips, for their collegiality and thoughtful discussions about this work. We are indebted to S. Park and K. Nguyen for providing the administrative assistance that allows us to focus on our research.

\section{LITERATURE CITED}

Abeysekera, R. M., Newcomb, W., Silvester, W. B., and Torrey, J. G. 1990. A freeze-fracture electron microscopic study of Frankia in root nodules of Alnus incana grown at three oxygen tensions. Can. J. Microbiol. 36: 97-108.

Althabegoiti, M. J., López-García, S. L., Piccinetti, C., Mongiardini, E. J., Pérez-Giménez, J., Quelas, J. I., Perticari, A., and Lodeiro, A. R. 2008. Strain selection for improvement of Bradyrhizobium japonicum competitiveness for nodulation of soybean. FEMS Microbiol. Lett. 282:115-123.

Barrière, Q., Guefrachi, I., Gully, D., Lamouche, F., Pierre, O., Fardoux, J., Chaintreuil, C., Alunni, B., Timchenko, T., Giraud, E., and Mergaert, P. 2017. Integrated roles of $\mathrm{BclA}$ and DD-carboxypeptidase 1 in Bradyrhizobium differentiation within NCR-producing and NCRlacking root nodules. Sci. Rep. 7:9063.

Belin, B. J., Busset, N., Giraud, E., Molinaro, A., Silipo, A., and Newman, D. K. 2018. Hopanoid lipids: From membranes to plant-bacteria interactions. Nat. Rev. Microbiol. 16:304-315.

Bonaldi, K., Gargani, D., Prin, Y., Fardoux, J., Gully, D., Nouwen, N., Goormachtig, S., and Giraud, E. 2011. Nodulation of Aeschynomene afraspera and A. indica by photosynthetic Bradyrhizobium sp. strain ORS285: The nod-dependent versus the nod-independent symbiotic interaction. Mol. Plant-Microbe Interact. 24:1359-1371.

Bradley, A. S., Swanson, P. K., Muller, E. E. L., Bringel, F., Caroll, S. M., Pearson, A., Vuilleumier, S., and Marx, C. J. 2017. Hopanoid-free Methylobacterium extorquens DM4 overproduces carotenoids and has widespread growth impairment. PLoS One 12:e0173323.

Caamal-Fuentes, E. E., Peraza-Sánchez, S. R., Torres-Tapia, L. W., and Moo-Puc, R. E. 2015. Isolation and identification of cytotoxic compounds from I, a Mayan medicinal plant. Molecules 20:13563-13574.

Chifundera, K. 2001. Contribution to the inventory of medicinal plants from the Bushi area, South Kivu Province, Democratic Republic of Congo. Fitoterapia 72:351-368.

Czernic, P., Gully, D., Cartieaux, F., Moulin, L., Guefrachi, I., Patrel, D., Pierre, O., Fardoux, J., Chaintreuil, C., Nguyen, P., Gressent, F., Da 
Silva, C., Poulain, J., Wincker, P., Rofidal, V., Hem, S., Barrière, Q., Arrighi, J.-F., Mergaert, P., and Giraud, E. 2015. Convergent evolution of endosymbiont differentiation in dalbergioid and inverted repeat-lacking clade legumes mediated by nodule-specific cysteine-rich peptides. Plant Physiol. 169:1254-1265.

Ehrhardt, D. W., Atkinson, E. M., and Long, S. R. 1992. Depolarization of alfalfa root hair membrane potential by Rhizobium meliloti Nod factors. Science 256:998-1000.

Gupta, R., Sharma, M., and Mittal, A. 2006. Effects of membrane tension on nanopropeller driven bacterial motion. J. Nanosci. Nanotechnol. 6: 3854-3862.

Hammad, Y., Nalin, R., Marechal, J., Fiasson, K., Pepin, R., Berry, A. M., Normand, P., and Domenach, A.-M. 2003. A possible role for phenyl acetic acid (PAA) on Alnus glutinosa nodulation by Frankia. Plant Soil 254:193-205.

Heckbert, P. S. 1994. Graphics gems IV. AP Professional, Boston.

Keymer, A., and Gutjahr, C. 2018. Cross-kingdom lipid transfer in arbuscular mycorrhiza symbiosis and beyond. Curr. Opin. Plant Biol. 44: 137-144.

Komaniecka, I., Choma, A., Mazur, A., Duda, K. A., Lindner, B., Schwudke, D., and Holst, O. 2014. Occurrence of an unusual hopanoid-containing lipid A among lipopolysaccharides from Bradyrhizobium species. J. Biol. Chem. 289:35644-35655.

Kulkarni, G., Busset, N., Molinaro, A., Gargani, D., Chaintreuil, C., Silipo, A., Giraud, E., and Newman, D. K. 2015. Specific hopanoid classes differentially affect free-living and symbiotic states of Bradyrhizobium diazoefficiens. MBio 6:e01251-15.

Ledermann, R., Bartsch, I., Müller, B., Wülser, J., and Fischer, H.-M. 2018. A functional general stress response of Bradyrhizobium diazoefficiens is required for early stages of host plant infection. Mol. Plant-Microbe Interact. 31:537-547.

Ledermann, R., Bartsch, I., Remus-Emsermann, M. N., Vorholt, J. A., and Fischer, H.-M. 2015. Stable fluorescent and enzymatic tagging of Bradyrhizobium diazoefficiens to analyze host-plant infection and colonization. Mol. Plant-Microbe Interact. 28:959-967.

Lee, C. K., de Anda, J., Baker, A. E., Bennett, R. R., Luo, Y., Lee, E. Y., Keefe, J. A., Helali, J. S., Ma, J., Zhao, K., Golestanian, R., O’Toole, G. A., and Wong, G. C. L. 2018. Multigenerational memory and adaptive adhesion in early bacterial biofilm communities. Proc. Natl. Acad. Sci. U.S.A. 115:4471-4476.

Lei, Z.-Y., Chen, J.-J., Cao, Z.-J., Ao, M.-Z., and Yu, L.-J. 2019. Efficacy of Aeschynomene indica Linn leaves for wound healing and isolation of active constituent. J. Ethnopharmacol. 228:156-163.

Nagy, A., Mowery, J., Bauchan, G. R., Wang, L., Nichols-Russell, L., and Nou, X. 2015. Role of extracellular structures of Escherichia coli O157: $\mathrm{H} 7$ in initial attachment to biotic and abiotic surfaces. Appl. Environ. Microbiol. 81:4720-4727.

Neubauer, C., Dalleska, N. F., Cowley, E. S., Shikuma, N. J., Wu, C.-H., Sessions, A. L., and Newman, D. K. 2015. Lipid remodeling in Rhodopseudomonas palustris TIE-1 upon loss of hopanoids and hopanoid methylation. Geobiology 13:443-453.

Ochs, D., Kaletta, C., Entian, K., Beck-sickinger, A., and Porallal, K. 1992. Cloning, expression, and sequencing of squalene-hopene cyclase, a key enzyme in triterpenoid metabolism. J. Bacteriol. 174:298-302.

Parsons, R., Silvester, W. B., Harris, S., Gruijters, W. T., and Bullivant, S. 1987. Frankia vesicles provide inducible and absolute oxygen protection for nitrogenase. Plant Physiol. 83:728-731.

Persat, A. 2017. Bacterial mechanotransduction. Curr. Opin. Microbiol. 36:1-6.

Petrova, O. E., and Sauer, K. 2012. Sticky situations: Key components that control bacterial surface attachment. J. Bacteriol. 194:2413-2425.

Pini, F., East, A. K., Appia-Ayme, C., Tomek, J., Karunakaran, R., Mendoza-Suárez, M., Edwards, A., Terpolilli, J. J., Roworth, J., Downie, J. A., and Poole, P. S. 2017. Bacterial biosensors for in vivo spatiotemporal mapping of root secretion. Plant Physiol. 174: 1289-1306.
Regensburger, B., and Hennecke, H. 1983. RNA polymerase from Rhizobium japonicum. Arch. Microbiol. 135:103-109.

Ricci, J. N., Coleman, M. L., Welander, P. V., Sessions, A. L., Summons, R. E., Spear, J. R., and Newman, D. K. 2014. Diverse capacity for 2methylhopanoid production correlates with a specific ecological niche. ISME J. 8:675-684.

Richards, F. J. 1959. NA flexible growth function for empirical use. J. Exp. Bot. 10:290-301.

Sáenz, J. P., Grosser, D., Bradley, A. S., Lagny, T. J., Lavrynenko, O., Broda, M., and Simons, K. 2015. Hopanoids as functional analogues of cholesterol in bacterial membranes. Proc. Natl. Acad. Sci. U.S.A. 112 : 11971-11976.

Schindelin, J., Arganda-Carreras, I., Frise, E., Kaynig, V., Longair, M., Pietzsch, T., Preibisch, S., Rueden, C., Saalfeld, S., Schmid, B., Tinevez, J. Y., White, D. J., Hartenstein, V., Eliceiri, K., Tomancak, P., and Cardona, A. 2012. Fiji: An open-source platform for biological-image analysis. Nat. Methods 9:676-682.

Schmerk, C. L., Welander, P. V., Hamad, M. A., Bain, K. L., Bernards, M. A., Summons, R. E., and Valvano, M. A. 2015. Elucidation of the Burkholderia cenocepacia hopanoid biosynthesis pathway uncovers functions for conserved proteins in hopanoid-producing bacteria. Environ. Microbiol. 17:735-750.

Schneider, C. A., Rasband, W. S., and Eliceiri, K. W. 2012. NIH image to ImageJ: 25 years of image analysis. Nat. Methods 9:671-675.

Silipo, A., Vitiello, G., Gully, D., Sturiale, L., Chaintreuil, C., Fardoux, J., Gargani, D., Lee, H.-I., Kulkarni, G., Busset, N., Marchetti, R., Palmigiano, A., Moll, H., Engel, R., Lanzetta, R., Paduano, L., Parrilli, M., Chang, W.-S., Holst, O., Newman, D. K., Garozzo, D., D’Errico, G., Giraud, E., and Molinaro, A. 2014. Covalently linked hopanoid-lipid A improves outer-membrane resistance of a Bradyrhizobium symbiont of legumes. Nat. Commun. 5:5106.

Siryaporn, A., Kuchma, S. L., O’Toole, G. A., and Gitai, Z. 2014. Surface attachment induces Pseudomonas aeruginosa virulence. Proc. Natl. Acad. Sci. U.S.A. 111:16860-16865.

Somado, E. A., Becker, M., Kuehne, R. F., Sahrawat, K. L., and Vlek, P. L. G. 2003. Combined effects of legumes with rock phosphorus on rice in West Africa. Agron. J. 95:1172.

Sugiyama, A., and Yazaki, K. 2012. Root exudates of legume plants and their involvement in interactions with soil microbes. Pages 27-48 in: Secretions and Exudates in Biological Systems. Signaling and Communication in Plants, Vol. 12. J. Vivanco and F. Baluška. eds. Springer, Berlin.

Swapna, M. M., Prakashkumar, R., Anoop, K. P., Manju, C. N., and Rajith, N. P. 2011. A review on the medicinal and edible aspects of aquatic and wetland plants of India. J. Med. Plants Res. 5:7163-7176.

Syrén, P.-O., Henche, S., Eichler, A., Nestl, B. M., and Hauer, B. 2016. Squalene-hopene cyclases-evolution, dynamics and catalytic scope. Curr. Opin. Struct. Biol. 41:73-82.

Szparaga, A., and Kocira, S. 2018. Generalized logistic functions in modelling emergence of Brassica napus L. PLoS One 13:e0201980.

Vilcheze, C., Llopiz, P., Neunlist, S., Poralla, K., and Rohmer, M. 1994 Prokaryotic triterpenoids: New hopanoids from the nitrogen-fixing bacteria Azotobacter vinelandii, Beijerinckia indica and Beijerinckia mobilis. Microbiology 140:2749-2753.

Welander, P. V., Coleman, M. L., Sessions, A. L., Summons, R. E., and Newman, D. K. 2010. Identification of a methylase required for 2methylhopanoid production and implications for the interpretation of sedimentary hopanes. Proc. Natl. Acad. Sci. U.S.A. 107:85378542 .

Welander, P. V, Doughty, D. M., Wu, C., Mehay, S., and Roger, E. 2012. Identification and characterization of Rhodopseudomonas palustris TIE-1 hopanoid biosynthesis mutants. Geobiology 10: 163-177.

Wu, C.-H., Bialecka-Fornal, M., and Newman, D. K. 2015. Methylation at the $\mathrm{C}-2$ position of hopanoids increases rigidity in native bacterial membranes. eLife 4:4. 